\title{
Model of the Jovian magnetic field topology constrained by the Io auroral emissions
}

\author{
S. L. G. Hess, ${ }^{1}$ B. Bonfond, ${ }^{2}$ P. Zarka, ${ }^{3}$ and D. Grodent ${ }^{2}$ \\ Received 4 November 2010; revised 20 January 2011; accepted 18 February 2011; published 24 May 2011.
}

[1] The determination of the internal magnetic field of Jupiter has been the object of many studies and publications. These models have been computed from the Pioneer, Voyager, and Ulysses measurements. Some models also use the position of the Io footprints as a constraint: the magnetic field lines mapping to the footprints must have their origins along Io's orbit. The use of this latter constraint to determine the internal magnetic field models greatly improved the modeling of the auroral emissions, in particular the radio ones, which strongly depends on the magnetic field geometry. This constraint is, however, not sufficient for allowing a completely accurate modeling. The fact that the footprint field line should map to a longitude close to Io's was not used, so that the azimuthal component of the magnetic field could not be precisely constrained. Moreover, a recent study showed the presence of a magnetic anomaly in the northern hemisphere, which has never been included in any spherical harmonic decomposition of the internal magnetic field. We compute a decomposition of the Jovian internal magnetic field into spherical harmonics, which allows for a more accurate mapping of the magnetic field lines crossing Io, Europa, and Ganymede orbits to the satellite footprints observed in UV. This model, named VIPAL, is mostly constrained by the Io footprint positions, including the longitudinal constraint, and normalized by the Voyager and Pioneer magnetic field measurements. We show that the surface magnetic fields predicted by our model are more consistent with the observed frequencies of the Jovian radio emissions than those predicted by previous models.

Citation: Hess, S. L. G., B. Bonfond, P. Zarka, and D. Grodent (2011), Model of the Jovian magnetic field topology constrained by the Io auroral emissions, J. Geophys. Res., 116, A05217, doi:10.1029/2010JA016262.

\section{Introduction}

[2] Jupiter generates the most intense planetary magnetic field of our solar system, with a magnetic moment of $4.2 \mathrm{G} /$ $R_{J}^{3}\left(1 R_{J}=71492 \mathrm{~km}\right)$, i.e., almost 18000 times Earth's field. This magnetic field creates a large magnetosphere around the planet, within which magnetic fields, internal plasma and satellites interact. Field aligned currents are induced by these interactions, they follow the magnetic field lines and close in the Jovian ionosphere, where they give rise to intense auroral emissions. Hence, the locations of the auroras on top of the Jovian ionosphere must correspond to the footprints of the magnetic field lines mapping to the regions of the magnetosphere where the interactions take place. In the case of the magnetospheric interaction with a satellite, this correspondence can be verified with a high accuracy, since the interaction is highly localized and the position of the satellite precisely known.

${ }^{1}$ LASP, University of Colorado at Boulder, Boulder, Colorado, USA.

${ }^{2}$ LPAP, Université de Liège, Liège, Belgium.

${ }^{3}$ LESIA, Observatoire de Paris-CNRS, Meudon, France.

Copyright 2011 by the American Geophysical Union. 0148-0227/11/2010JA016262
[3] The most intense satellite-driven auroras are those due to Io's interaction with the Jovian magnetosphere, which injects a power of about $10^{12}$ Watts per hemisphere in the magnetosphere. The Io-related auroral emissions cover a large range of the electromagnetic spectrum, from lowfrequency radio [Bigg, 1964] to X-rays [BranduardiRaymont et al., 2008] through UV [Clarke et al., 1996; Prangé et al., 1996] and Infrared [Connerney et al., 1993]. The UV auroral footprints of Io consist of a main spot, secondary spots and an extended tail. This substructure of the footprint is interpreted as being due to the Alfvénic current system at Io [Neubauer, 1980; Saur, 2004] (also sketched in Figure 1): As the current-carrying Alfvén waves generated at Io travel toward Jupiter, they are partially reflected and dispersed, which produces the observed spots [Hess et al., 2010b; Jacobsen et al., 2007], and causes the formation of a quasi-steady state current system powering the tail [Hill and Vasyliünas, 2002; Ergun et al., 2009]. The main spot is related to the most direct trajectory of the current-carrying Alfvén waves, hence the associated magnetic field lines should map close to Io's position.

[4] Many studies of the magnetic mapping of Io to the planet and its comparison with the positions of Io's footprints observed in UV and Infrared have been performed [Connerney et al., 1998; Grodent et al., 2008a; Bonfond et al., 

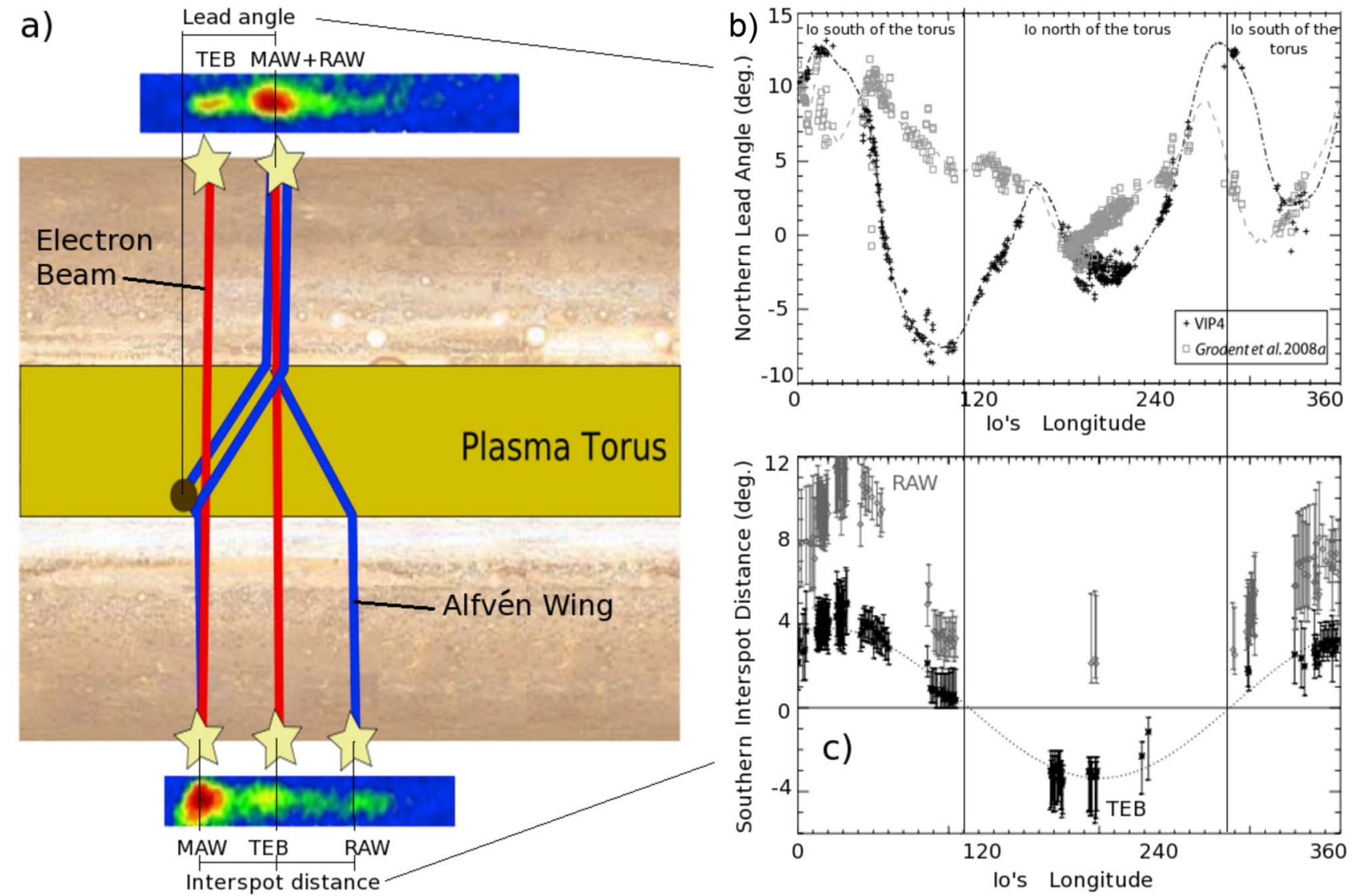

Figure 1. (a) Sketch of the UV aurora generation by the Io-Jupiter interaction. Main Alfvén wing (MAW) and reflected Alfvén wing (RAW) spots are generated by electrons locally accelerated by an Alfvén wave (reflected for the RAW) whereas TEB spots are generated by an electron beam accelerated in the opposite hemisphere. (b) Measurements of the lead angle between the location of the observed northern MAW and the one predicted by the VIP4 and the Grodent et al. [2008a] model. Vertical lines indicate the crossing of the torus center (centrifugal equator). (c) Positions of the southern TEB and RAW spots relative to the MAW spot (adapted from Bonfond et al. [2008, 2009]).

2009], which have led to the determination of new internal magnetic field models [Connerney et al., 1998; Connerney, 2007; Grodent et al., 2008a]. However, none of these models allowed a correct longitudinal mapping of the Io footprints: Studies by Bonfond et al. [2009] in the UV and by Hess et al. [2010a] in radio showed a large (up to $10^{\circ}$ ) longitude difference between the predicted and observed positions of the Io footprints. These studies are discussed in more detail in section 2 .

[5] The purpose of the present paper is to determine a model of the topology of the magnetic field lines at Jupiter, which permits one to accurately map the observed Io UV footprints to Io's position. Our model is mostly constrained by the mapping of auroral emissions to the equatorial plane (namely the UV observations of the Io footprints at the surface of Jupiter mapped to Io), whereas the existing internal magnetic field models of Jupiter are based on the magnetic field measurements performed by spacecraft flying by the planet, generally close to the equatorial plane. The uncertainties on the spherical harmonic coefficients obtained from these two approaches are different. In particular, our method does not separate the uncertainties on each spherical harmonic coefficient as well as the methods based on the inversion of the magnetic field measurements do. This should be remembered before using it for some studies, in particular studies of the Jovian interior.

[6] However, we show that our internal magnetic field model describes fairly well (with an accuracy lower but still comparable with previous models) the magnetic field lines crossing the equatorial plane in the outer part of the inner magnetosphere and in the middle magnetosphere (that is farther than $\sim 4 R_{J}$ from the planet center). In particular, the magnetic field model we present in this study accurately predicts the location of the auroral emissions associated with Io, and the corresponding magnetic field strength at the Jovian surface. Moreover, we show that this magnetic field model also gives a better mapping of the Europa and Ganymede northern footprints than the VIP4 model. Our model is an update to the VIP4 internal magnetic field model, which includes the modeling of the lowest orders of the magnetic Anomaly and corrects for the Longitudinal position of the magnetic field lines mapping to Io's orbit. Hence, we hereafter refer to it as the VIPAL magnetic field model. 
[7] In section 2, we present a brief summary of the previous modelings of Jupiter's magnetic field. The observational constraints on the magnetic field model are discussed in section 3. The method used to determine our magnetic field model, and the resulting magnetic field spherical harmonics coefficients are presented in sections 4 and 5 . The magnetic field model is then compared to observations in section 6, and its characteristics are discussed in section 7 .

\section{Magnetic Field Models}

[8] The magnetic field of Jupiter, as the magnetic fields of any other planet with the exception of the Earth, has first been modeled from the magnetic field measurements performed by spacecraft flying by the planet. In the case of Jupiter, the Pioneer 10 and 11 [M. H. Acuna and N. F. Ness, 1975], and Voyager 1 [Ness et al., 1979a] measurements provided the most important constraints on the magnetic field determination [Connerney, 1993]. Additional data were obtained by the Voyager 2 [Ness et al., 1979b], Ulysses [Connerney et al., 1996] and Galileo flyby, but imposed fewer constraints as a result of the larger closest approach distance of the spacecraft to Jupiter.

[9] Models of the Jovian magnetic field were built by performing an inversion of these measurements. The magnetospheric magnetic field $(B)$ can be split into a planetary internal magnetic field, which derives from a potential field $(V)$, and a magnetic perturbation $(b)$ owing to magnetospheric currents:

$$
B=-\nabla V+b
$$

The expression of the magnetospheric perturbation $(b)$ is derived from magnetospheric current observations and modeling, and is not precisely known [Connerney et al., 1981; Khurana, 1997; Khurana and Schwarzl, 2005]. Moreover this current is likely to change with conditions in the magnetosphere. These currents have a substantial impact on the magnetic field lines crossing the equatorial plane far from the planet, but can be neglected for field lines reaching less than a few Jovian radii. The footpaths associated with the orbit of Io are little affected (less than a degree) by the current sheet density [Grodent et al., 2008b]. This is consistent with the orbit of Io being in the inner magnetosphere $\left(\sim 5.95 R_{J}\right.$ from Jupiter center), where the total magnetic field is primarily controlled by the Jovian internal field. At larger distances, the influence of the current sheet grows and may become dominant in the middle magnetosphere: the poleward shift becomes substantial (a few degrees) for the footpath mapping to the orbit of Ganymede, $\sim 15 R_{J}$ from Jupiter center [Grodent et al., 2008b]. The potential field $V$ can hence be derived from the magnetic field measurements inside $\sim 10 R_{J}$. The potential field is usually expressed in term of spherical harmonics [Connerney, 1981]:

$$
V=\sum_{n} \sum_{m} \frac{P_{n}^{m}(\cos \theta)\left[g_{n}^{m} \cos (m \phi)+h_{n}^{m} \sin (m \phi)\right]}{r^{n+1}}
$$

where $P_{n}^{m}$ is the Schmidt-normalized Legendre function of degree $n$ and order $m$, and the $g_{n}^{m}$ and $h_{n}^{m}$ terms are the Schmidt coefficients describing the planet's internal mag- netic field, and $r$ is the distance relative to the planet's center, normalized to the Jovian equatorial radius.

[10] The most accurate magnetic field model constructed only using the inversion of magnetic field measurements is the O6 magnetic model [Connerney et al., 1996]. However, this model does not precisely map to the observed location of the Io footpaths. As a result, a new magnetic field model was developed to fit both the magnetic field measurements and the location of the Infrared footprints of Io: The VIP4 model [Connerney et al., 1998].

[11] However, since the creation of VIP4, the number of UV observations of the Io footprints considerably increased, with better spatial resolution than in infrared. The most recent and precise table of the Io footprints location versus Io orbital longitude has been published by Bonfond et al. [2009] from observations in the UV, and is included in Table 1. The northern Io footpath obtained from the VIP4 model does not map the observed UV footprints as well as it mapped the less accurately defined infrared ones (Figure 2). The southern Io footpath is nevertheless accurately modeled. A later model, the VIT4 internal magnetic model [Connerney, 2007], was developed using mostly the position of the Io footpaths and the azimuthal component of the magnetic field measured by Voyager. These Voyager measurements are necessary because the longitudinal correspondence between the observed footprint and Io was not used, and the only fit of the Io footpaths does not put sufficient constraints on the azimuthal component of the magnetic field.

[12] More recently, UV observations of the Europa and Ganymede footprints have also been performed [Clarke et al., 2002; Grodent et al., 2006, 2009]. Grodent et al. [2008a] give a table of the Europa and Ganymede footprint locations versus the longitude of the satellites, which is also included in Table 1. The VIP4 model does not map accurately the northern hemisphere footpaths of these satellites (Figure 3). In order to obtain a better fit of these footpaths, in particular the kink in the Io northern footpath near a longitude of $110^{\circ}$, Grodent et al. [2008a] proposed to add a magnetic anomaly modeled by a supplementary dipole and found to potential locations at a longitude of about $115^{\circ} \pm 5^{\circ}$ and a latitude of about $66^{\circ} \pm 2^{\circ}$ north. Two solutions were found, both with a dipole whose magnetic moment was $\sim 1 \%$ of the main dipolar moment. With this supplementary ad hoc dipole, Grodent et al. [2008a] were able to fit the Io, Europa and Ganymede northern footpaths. The southern ones, however, were not accounted for, and are not well mapped.

[13] Comparison of the longitudinal correspondence between the positions of the Io footprints modeled with VIP4 and the observed ones also showed that the azimuthal component of the magnetic field was not accurately described by these models [Bonfond et al., 2009] (see also Figure 4). This is not surprising, as the longitude was never used as an observational constraint to determine the magnetic field models [Connerney et al., 1998]. The absence of longitudinal constraints is a source of difficulties for modeling of the auroral emissions. In particular, the modeling of the Io-controlled radio emissions, which are affected by the relative geometry of the emission sources and the observer [Hess et al., 2008], cannot be properly performed without adding an ad hoc correction to the longitude of the magnetic field lines emitting in radio [Hess et al., 2010a]. Moreover, the modeling could only be performed for emissions coming 
Table 1. Positions of the Satellite Footprints Observed in UV as a Function of the Satellite System III Longitudes ${ }^{\mathrm{a}}$

\begin{tabular}{|c|c|c|c|c|c|c|c|c|c|c|c|c|}
\hline \multirow[b]{2}{*}{$\begin{array}{l}\text { Satellite } \\
\text { Long. } \\
\text { (deg) }\end{array}$} & \multicolumn{4}{|c|}{ Io } & \multicolumn{4}{|c|}{ Europa } & \multicolumn{4}{|c|}{ Ganymede } \\
\hline & $\begin{array}{l}\text { North } \\
\text { Long. } \\
\text { (deg) }\end{array}$ & North Lat. (deg) & $\begin{array}{l}\text { South } \\
\text { Long. } \\
\text { (deg) }\end{array}$ & $\begin{array}{l}\text { South } \\
\text { Lat. } \\
\text { (deg) }\end{array}$ & $\begin{array}{l}\text { North } \\
\text { Long. } \\
\text { (deg) }\end{array}$ & $\begin{array}{l}\text { North } \\
\text { Lat. } \\
\text { (deg) }\end{array}$ & $\begin{array}{l}\text { South } \\
\text { Long. } \\
\text { (deg) }\end{array}$ & $\begin{array}{l}\text { South } \\
\text { Lat. } \\
\text { (deg) }\end{array}$ & $\begin{array}{l}\text { North } \\
\text { Long. } \\
\text { (deg) }\end{array}$ & $\begin{array}{l}\text { North } \\
\text { Lat. } \\
\text { (deg) }\end{array}$ & $\begin{array}{l}\text { South } \\
\text { Long. } \\
\text { (deg) }\end{array}$ & $\begin{array}{l}\text { South } \\
\text { Lat. } \\
\text { (deg) }\end{array}$ \\
\hline 0 & -32.0 & 76.9 & 5.1 & -61.4 & & & 4.9 & -64.8 & & & 11.7 & -67.0 \\
\hline 10 & -22.6 & 78.5 & 14.1 & -61.0 & & & 12.6 & -64.0 & & & 23.3 & -66.4 \\
\hline 20 & -2.1 & 81.0 & 23.2 & -60.6 & & & 21.9 & -63.2 & & & 32.3 & -66.0 \\
\hline 30 & 32.7 & 82.7 & 32.2 & -60.1 & & & 31.7 & -62.6 & & & 37.9 & -65.9 \\
\hline 40 & 70.7 & 81.4 & 40.9 & -59.3 & & & 40.7 & -62.3 & 138.3 & 75.2 & 41.0 & -65.8 \\
\hline 50 & 96.9 & 77.0 & 49.2 & -58.5 & 123.7 & 77.1 & 48.2 & -62.3 & 138.9 & 74.4 & 43.5 & -65.7 \\
\hline 60 & 110.1 & 69.3 & 56.9 & -57.7 & 128.6 & 72.6 & 54.2 & -62.3 & 140.0 & 72.7 & 47.1 & -65.7 \\
\hline 70 & 118.6 & 64.3 & 64.1 & -57.3 & 133.1 & 66.7 & 59.0 & -62.5 & 141.7 & 69.0 & 52.5 & -65.7 \\
\hline 80 & 126.3 & 60.9 & 71.0 & -57.3 & 137.2 & 62.2 & 63.5 & -62.7 & 143.0 & 64.7 & 59.2 & -65.8 \\
\hline 90 & 132.6 & 57.9 & 77.8 & -57.7 & 140.5 & 59.5 & 68.2 & -63.0 & 144.6 & 61.9 & 66.4 & -66.2 \\
\hline 100 & 137.7 & 55.0 & 84.5 & -58.6 & 144.3 & 56.7 & 73.1 & -63.4 & 146.7 & 59.7 & 73.5 & -66.9 \\
\hline 110 & 142.1 & 52.6 & 91.3 & -60.0 & 148.2 & 54.7 & 78.2 & -64.0 & 149.2 & 58.0 & 80.4 & -68.0 \\
\hline 120 & 145.8 & 50.8 & 98.3 & -61.8 & 151.6 & 53.5 & 83.7 & -64.6 & 152.3 & 56.2 & 87.3 & -69.3 \\
\hline 130 & 150.0 & 49.5 & 105.5 & -63.8 & 155.1 & 52.5 & 90.0 & -65.4 & 155.5 & 54.9 & 93.6 & -70.7 \\
\hline 140 & 154.5 & 48.8 & 113.2 & -66.0 & 158.5 & 51.9 & 98.2 & -66.7 & 158.8 & 54.2 & 98.7 & -72.0 \\
\hline 150 & 158.4 & 48.6 & 121.8 & -68.1 & 161.7 & 51.6 & 109.8 & -68.8 & 162.2 & 53.6 & 102.2 & -72.9 \\
\hline 160 & 162.7 & 48.7 & 131.6 & -70.2 & 165.1 & 51.5 & 125.9 & -71.9 & 165.5 & 53.5 & 105.2 & -73.7 \\
\hline 170 & 168.1 & 48.9 & 143.3 & -72.1 & 168.8 & 51.5 & 146.8 & -75.8 & 169.0 & 53.5 & 110.7 & -75.1 \\
\hline 180 & 173.5 & 49.5 & 156.8 & -73.9 & 172.6 & 51.9 & 171.3 & -79.4 & 172.3 & 53.6 & 122.6 & -77.9 \\
\hline 190 & 178.4 & 50.4 & 172.1 & -75.6 & 176.5 & 52.5 & 197.2 & -81.1 & 175.6 & 54.0 & 143.9 & -81.0 \\
\hline 200 & 183.2 & 51.7 & 188.6 & -76.8 & 180.6 & 53.4 & 221.5 & -80.8 & 179.3 & 54.8 & 174.4 & -82.4 \\
\hline 210 & 188.5 & 53.3 & 205.4 & -76.8 & 184.6 & 54.5 & 241.5 & -79.5 & 183.2 & 55.8 & 210.6 & -83.7 \\
\hline 220 & 194.3 & 55.0 & 221.7 & -76.2 & 188.5 & 55.6 & 255.6 & -78.1 & 187.1 & 56.8 & 246.2 & -82.7 \\
\hline 230 & 200.5 & 56.6 & 236.6 & -75.6 & 192.8 & 57.0 & 264.2 & -77.2 & 191.1 & 57.8 & 275.5 & -80.1 \\
\hline 240 & 207.5 & 58.5 & 250.1 & -75.0 & 196.9 & 58.3 & 269.2 & -76.6 & 195.3 & 59.0 & 295.2 & -77.8 \\
\hline 250 & 214.6 & 60.4 & 262.0 & -74.2 & 201.6 & 59.6 & 273.2 & -76.2 & 199.8 & 60.2 & 305.7 & -76.2 \\
\hline 260 & 220.8 & 61.6 & 272.8 & -73.0 & 206.4 & 60.7 & 278.9 & -75.5 & 205.0 & 61.6 & 310.6 & -75.4 \\
\hline 270 & 228.0 & 63.0 & 282.9 & -71.7 & & & 287.9 & -74.4 & 210.5 & 62.9 & 314.0 & -74.8 \\
\hline 280 & 238.8 & 65.1 & 292.6 & -70.4 & & & 300.1 & -72.9 & 216.5 & 64.8 & 318.7 & -74.0 \\
\hline 290 & 252.1 & 67.7 & 302.2 & -69.0 & & & 314.1 & -71.1 & 222.5 & 66.8 & 324.8 & -72.9 \\
\hline 300 & 265.4 & 69.8 & 311.7 & -67.8 & & & 327.7 & -69.3 & 229.0 & 68.7 & 330.9 & -71.8 \\
\hline 310 & 278.4 & 70.9 & 321.0 & -66.5 & & & 338.9 & -67.9 & 235.9 & 70.6 & 335.8 & -70.9 \\
\hline 320 & 290.6 & 71.6 & 330.0 & -65.2 & & & 346.9 & -66.9 & 243.0 & 72.4 & 339.5 & -70.3 \\
\hline 330 & 301.5 & 72.5 & 338.8 & -64.0 & & & 352.0 & -66.2 & 250.6 & 74.9 & 343.6 & -69.7 \\
\hline 340 & 311.6 & 73.9 & 347.5 & -62.9 & & & 355.5 & -65.8 & & & 350.2 & -68.9 \\
\hline 350 & 320.7 & 75.5 & 356.3 & -62.0 & & & 359.4 & -65.4 & & & 359.9 & -67.8 \\
\hline
\end{tabular}

${ }^{\mathrm{a}}$ Io data are adapted from Bonfond et al. [2009]. Europa and Ganymede data for the northern hemisphere are adapted from Grodent et al. [2008a]. The errors on each measurement are not indicated and must be found in the cited papers [Bonfond et al., 2009; Grodent et al., 2008a]. However, the error is mainly due to projection problems and varies with the satellite footprint latitude. The typical errors on longitude and latitude are indicated as a function of the satellite footprint latitude [Grodent et al., 2008a].

from the southern hemisphere: the frequency of the radio emissions is close to the local electron cyclotron frequency, so that the maximum observed radio frequency is related to the magnetic field strength at the top of Jupiter's ionosphere. The study of the maximum frequency versus Io's longitude showed that many of the northern emissions were observed with a frequency corresponding to magnetic field strengths far larger than those predicted by the magnetic field models (see discussion in section 3.5), which is an unphysical result.

[14] Table 2 summarizes the main characteristics (constraints and observations reproduced) of each of these models.

\section{Observational Constraints}

[15] Many observations relevant for our studies have been performed, which constrain the position of the Io footprint (UV), the morphology of the magnetic field lines (radio) and the magnetic field strength (magnetic field measurements and radio observations).In this section, we discuss the constraints brought by these observations.

\subsection{Morphology of the Io Footprints}

[16] The interaction between the magnetosphere of Jupiter and its three innermost Galilean satellites generates intense UV emissions in the Jovian ionosphere. The Io footprint is the most intense and best resolved satellite footprint. It is composed of: A main spot (main Alfvén wing or MAW spot), secondary spots (reflected Alfvén wing or RAW spots) and a diffuse tail which has an extent of several tens of degrees [Connerney and Satoh, 2000; Gérard et al., 2006]. These features are thought to be a consequence of the Aflvénic interaction between Io and Jupiter: The motion of Io relative to Jupiter's magnetic field lines and the corotating plasma excites Alfvén wave packets in Io's vicinity [Neubauer, 1980; Saur et al., 2004]. These wave packets propagate from Io to Jupiter, forming the so-called Alfvén wings. On their way, the Alfvén waves encounter density and magnetic field strength variations, which result in the 


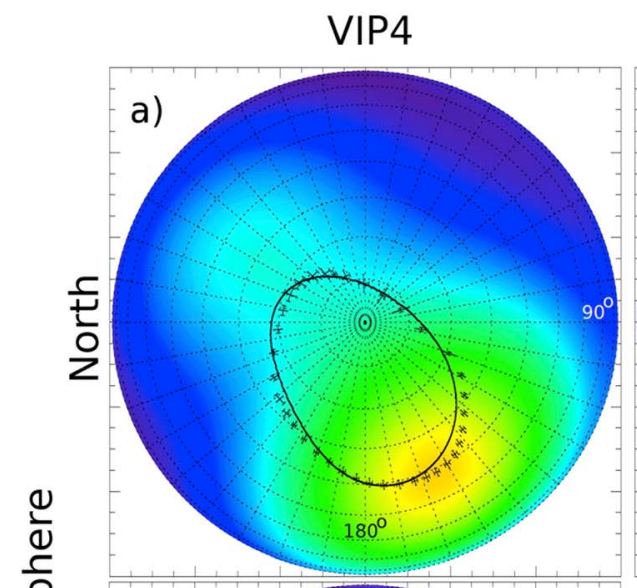

Grodent et al., 2008a
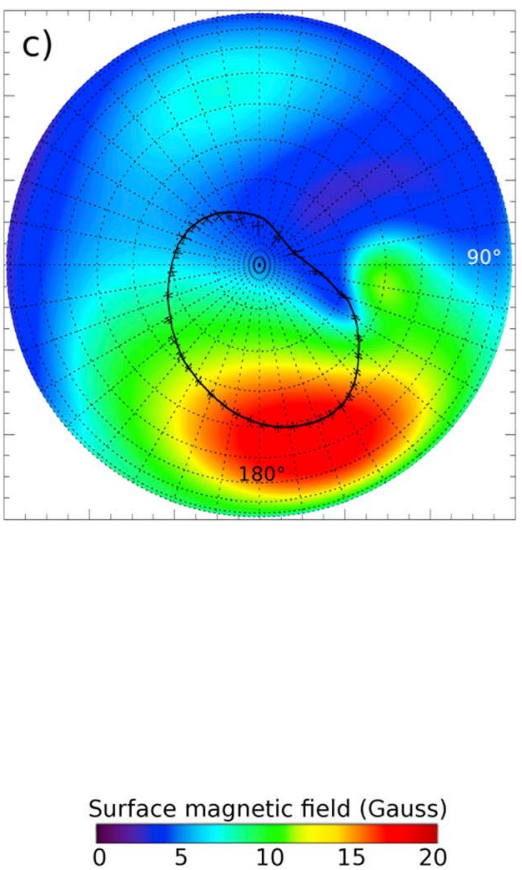
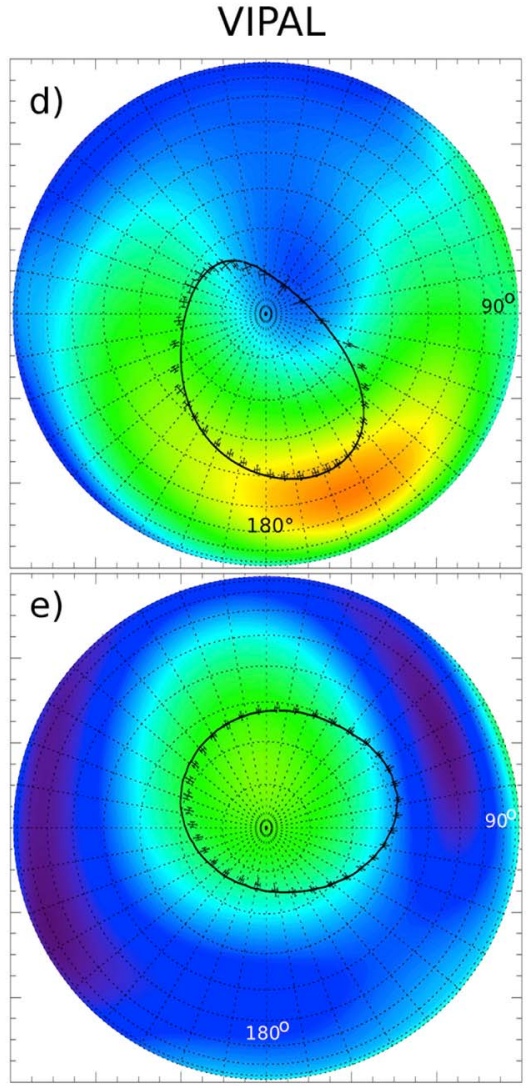

Figure 2. Surface field, observed Io's UV footprint (crosses), and modeled Io's footpaths (continuous line). (a) VIP4 model, northern hemisphere; (b) VIP4 model, southern hemisphere; (c) Grodent et al. [2008a] model, northern hemisphere; (d) VIPAL model, northern hemisphere; and (e) VIPAL model, southern hemisphere.

partial reflection of the waves. This leads to the existence of a main Alfvén wing (MAW), which corresponds to the most direct trajectory of the Alfvén waves, and reflected Alfvén wings (RAWs) which corresponds to the trajectories of the reflected Alfvén waves.

[17] The orbit of Io is immersed in a dense plasma torus (see review by Thomas et al. [2004]), due to the volcanic activity of the satellite. In the torus, the velocity of the Alfvén waves is low (down to $200 \mathrm{~km} / \mathrm{s}$ ). Hence, the Alfvén waves need an average 8 minutes to escape the torus, a time during which the magnetic field line carrying them move by $\sim 4^{\circ}$ in longitude [Delamere et al., 2003]. The main Alfvén wing thus reaches the planet on a magnetic field line which does not map exactly to Io's position but is slightly shifted in longitude. This shift is called equatorial lead angle.

[18] The RAW spots have mostly been observed in UV [Gérard et al., 2006; Bonfond et al., 2008, 2009], although a RAW observation has been reported in IR [Connerney and Satoh, 2000]. As the position of Io relative to the center of the torus varies with longitude, the shifts of the RAW spots relative to the MAW and to each other vary too. When Io is on a border of the torus the first RAW merges with the MAW spot, whereas the second RAW is at its maximal shift relative to the MAW $\left(\sim 12^{\circ}\right)$ [Bonfond et al., 2009]. This property will be used in section 3.3 to determine the equa- torial longitude of the magnetic field line mapped to the observed UV footprints.

[19] A third class of spot has been observed in UV [Bonfond et al., 2008]: the transhemispheric electron beam (TEB) spot. It is sometimes called a precursor, but as it can sometimes trail the MAW spot this name is inappropriate. A detailed description of the characteristics of this spot can be found in the work by Bonfond et al. [2008, 2009]. The TEB spot has a longitudinal shift relative to the MAW spot varying from about $-4^{\circ}$ to $+4^{\circ}$. The fact that this spot may precede the MAW spot implies that it cannot be due to Alfvén wave reflection. It has first been suggested by Bonfond et al. [2008] that this spot may be due to electron beams accelerated in a given hemisphere above the MAW spot, which precipitate in the opposite hemisphere. This suggestion was further supported by the theoretical approach by Hess et al. [2010b], which showed that the MAW is most probably created by the electron acceleration by Alfvén waves, which accelerate electrons both toward Jupiter and antiplanetward.

[20] Figure 1a shows a sketch of the Alfvén waves trajectories, including the reflected waves, as well as the position of the auroral spots they generate. Figure $1 \mathrm{~b}$ shows the measurements of the equatorial lead angle in the southern hemisphere, deduced from the VIP4 model 

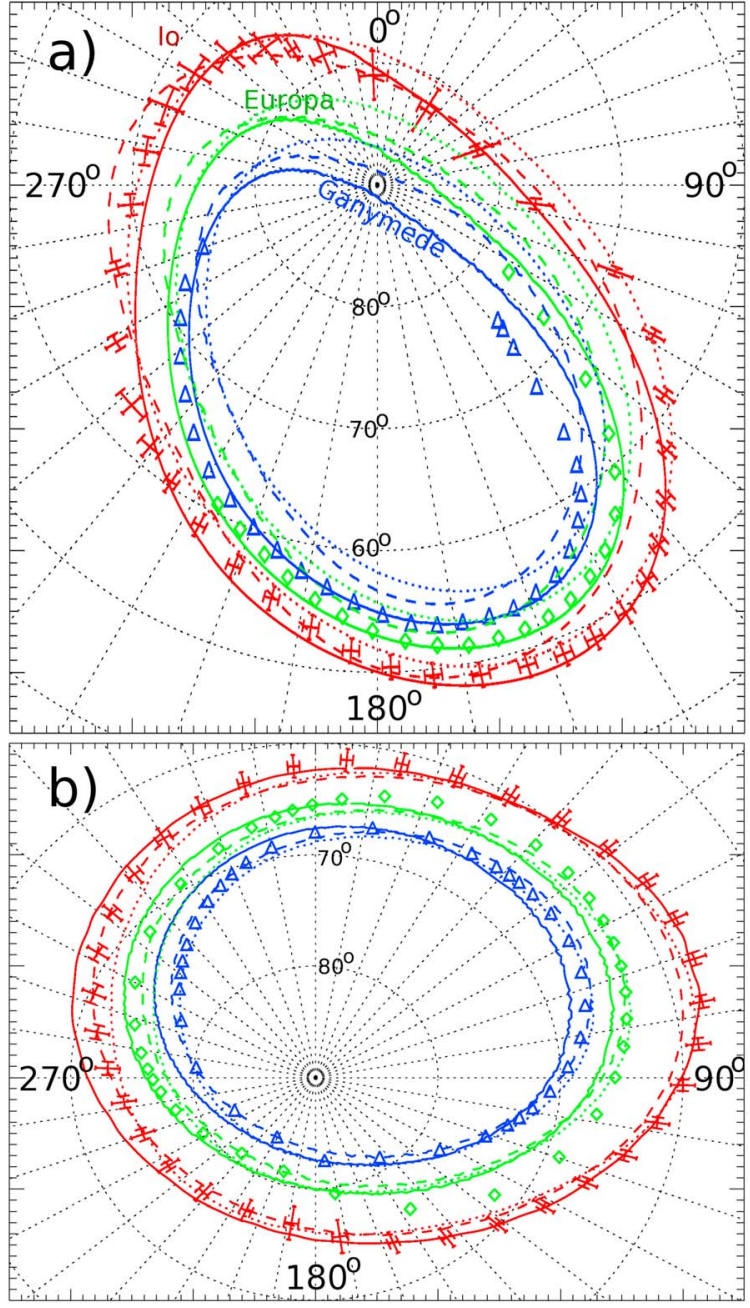

Figure 3. Observed footprints of Io [from Bonfond et al., 2009] (with error bars), Europa [from Grodent et al., 2008a] (diamonds), and Ganymede [from Grodent et al., 2008a] (triangles). (a) Northern hemisphere and (b) southern hemisphere. Solid lines stand for the footpath from the VIPAL model, dashed lines stand for the footpath from the VIP4 model, and dotted lines stand for the footpath from the VIT4 model.

[Bonfond et al., 2009]. Figure 1c shows the longitude difference between the main spot and the TEB and RAW spots in the southern hemisphere, versus Io's longitude, as measured by Bonfond et al. [2009].

\subsection{Location of the Satellite UV Footprints}

[21] The UV spots are assumed to map to the equatorial plane location of the interaction region surrounding the satellite, with a slight shift in longitude due to the finite propagation time of the Alfvén waves. We use this footprint correspondence to develop our magnetic field model.

[22] The best data set for measuring the location of the satellite footprints currently comes from the HST observations of the $\mathrm{H}_{2}$ and $\mathrm{H}$ emissions in the far ultraviolet domain. More precisely, these data sets are obtained from the Space Telescope Imaging Spectrograph (STIS) and the Advanced Camera for Surveys (ACS) instruments of the HST. Depending on the observing geometry, the position of the satellite footprints can be determined with an accuracy ranging from $0.5^{\circ}$ to $\sim 8^{\circ}$ both in longitude and latitude. The Io footprint itself is a thin curtain $<200 \mathrm{~km}$ wide [Bonfond, 2010], composed of at least three spots and a trailing tail extending up to $100^{\circ}$ in the downstream direction. The peak altitude of these different structures ranges from 700 to $900 \mathrm{~km}$ above the planetary surface [Bonfond et al., 2009; Bonfond, 2010]. Inside the footprint, the brightest MAW spot corresponds to the most direct trajectory of the currentcarrying Alfév waves between Io and Jupiter. The MAW span for $\sim 850 \mathrm{~km}$ along the Io footpath, which corresponds to approximately $1^{\circ}-2^{\circ}$ in longitude [Gérard et al., 2006; Bonfond, 2010]. Many observations of the UV footprint have been performed. The averaged positions of Io's main UV spot, versus Io's orbital longitude, have been published by Grodent et al. [2008a] for the northern hemisphere and by Bonfond et al. [2009] for the southern one.

[23] Grodent et al. [2008a] provide a list of Europa's and Ganymede's footprint positions versus satellite orbital longitudes restricted to the northern hemisphere regions visible from Earth orbit and where the footprints appear detached from the nearby main auroral oval emission (which is related to the corotation breakdown $20-30 R_{J}$ from Jupiter). We have performed similar measurements for the southern hemisphere. These data are summarized in Table 1.

[24] Figure 3 shows the position of the observed satellite footprints and the mapping on Jupiter of the magnetic field lines connected to the Io, Europa and Ganymede orbits performed with the VIP4 model (dashed lines). A model of the contribution of the current sheet to the magnetic field is needed to model the Europa and Ganymede footprints. In this paper we mapped the Europa and Ganymede footpaths using the Connerney et al. [1981] current sheet model. More recent and detailed models of the current sheet exist [Khurana, 1997; Khurana and Schwarzl, 2005]. These models improve mostly the current sheet description in the outer magnetosphere, and do not improve the magnetic field line mapping for regions that are close to the planet. The footpaths mapped with the VIP4 plus current sheet model match well the observed ones in the southern hemisphere, but no longer fit the most recently observed UV northern footpath, in particular those of Europa and Ganymede.

[25] We measure the accuracy of the mapping of the northern and southern Io footprints by measuring the mean distance between the footprints observed in UV and those given by our model. There are many ways to define the modeled footprints. In the present paper, we define a modeled footprint as the point along the modeled footpath (obtained by mapping the satellite orbit on the planet surface, regardless of longitudes), which is the closest to an observed UV footprint (Figure 5). With this definition, we are sure that the modeled footprint maps to the satellite orbit. The accuracy of the modeling is measured by two values: the angular distance on the planet surface between the modeled and observed footprints, and the difference between the expected equatorial longitude of the field line and the longitude at which the modeled footprints is mapped in the equatorial plane. The latter is discussed in section 3.3. 

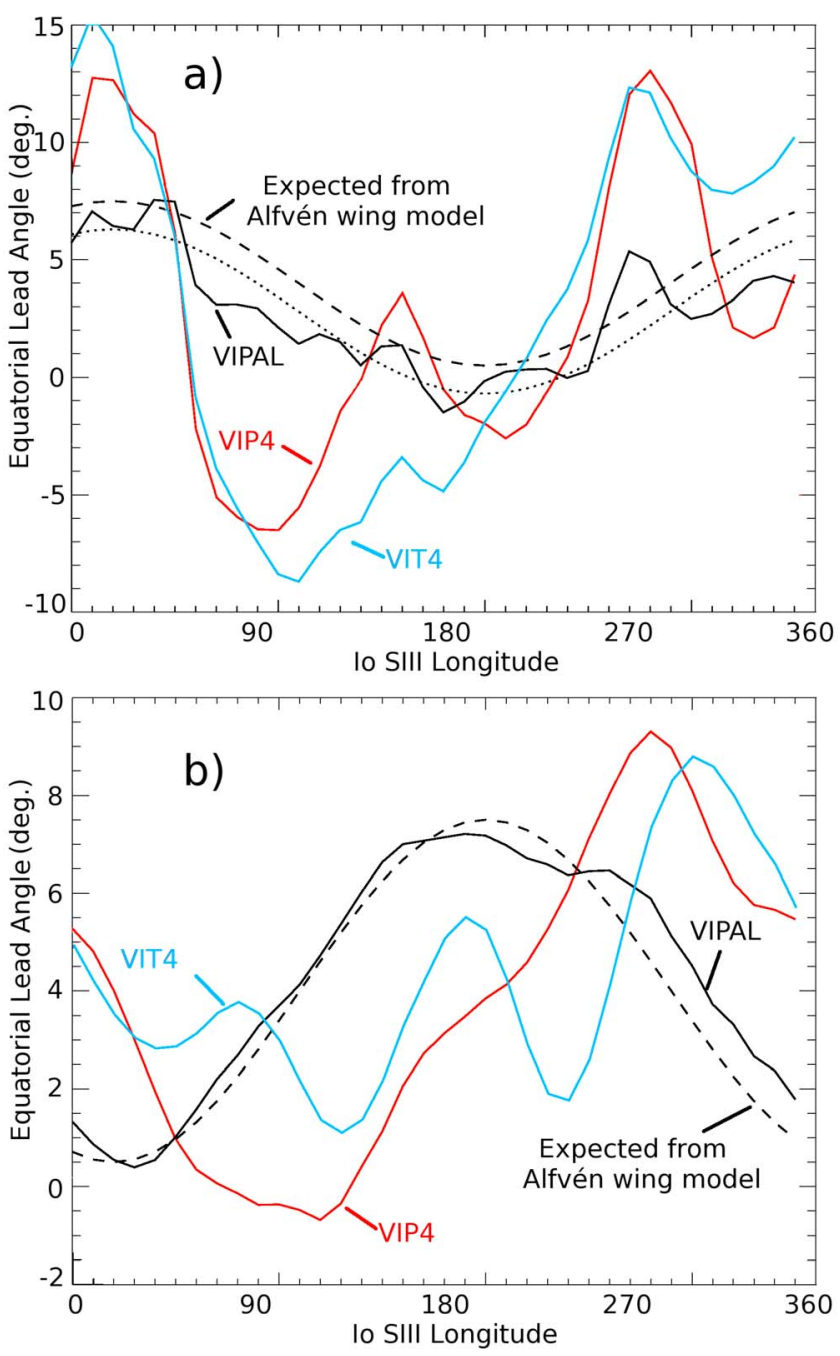

Figure 4. Equatorial lead angles. The dashed lines show the expected amplitude of the equatorial lead angle variation, based on the Alfvén wing model. (a) In the northern hemisphere field lines, VIP4 model (red line) and VIPAL model (black line). The dotted line is the expected variation shifted by $1.2^{\circ}$ to match the mean value of the lead angle in the northern hemisphere. (b) For the southern hemisphere field lines, VIP4 model (red line), VIT4 (blue line), and VIPAL model (black line).

[26] The mean angular distance between the modeled and observed footprints $\left(\Delta_{N / S}\right)$ is computed independently for each hemisphere:

$$
\begin{aligned}
& \Delta_{N / S}^{2}=\frac{4}{N_{\text {observations }}} \sum_{\text {observations }} \arcsin ^{2} \\
& \cdot\left(\sqrt{\sin ^{2}\left(\frac{\theta_{\text {obs }}-\theta_{\text {mod }}}{2}\right)+\sin \left(\theta_{\text {obs }}\right) \sin \theta_{\text {mod }} \sin ^{2}\left(\frac{\lambda_{\text {obs }}-\lambda_{\text {mod }}}{2}\right)}\right)
\end{aligned}
$$

where $\theta_{o b s}$ and $\theta_{\text {mod }}$ are the colatitudes of the observed and modeled footprints, respectively, and $\lambda_{o b s}$ and $\lambda_{\text {mod }}$ are the longitudes of the observed and modeled footprints at the surface of the planet, respectively. The mapping of Io's footprints with the VIP4 internal magnetic field model gives mean angular distances of $1.5^{\circ}$ in the northern hemisphere, and $0.9^{\circ}$ in the southern one, these distances are smaller than $1^{\circ}$ for the VIT4 model, and the model of Grodent et al. [2008a] allows to map the Io footpath in the northern hemisphere with a mean error smaller than $0.5^{\circ}$. Note that, even though we took into account the oblateness of the planet in our mapping, we did not take it into account for computing the distance to the observed spots $\Delta_{N / S}$. The radius does not vary by much along the footpaths, so that the spatial distance can be approximated as proportional to the angular distance.

\subsection{Equatorial Longitude of the Magnetic Field Lines}

[27] We want our magnetic field model to give the correct longitudinal mapping of the Io main spot. We mentioned above that this spot is not thought to be the footprint of a magnetic field line mapping to the position of Io, but a few degrees downstream of the satellite, due to the finite travel time of the current carrying Alfvén waves. This longitudinal difference, called lead angle, is not constant: The Io plasma torus orbits in the centrifugal equator plane, which is inclined by $\sim 7^{\circ}$ relative to the jovigraphic equatorial plane in which Io orbits. Thus the distance the Alfvén waves take to traverse the torus vary with the orbital phase of Io: when Io is located north of the torus (near $220^{\circ}$ ), the Alfvén wave propagating toward the northern hemisphere of Jupiter has a short distance to travel in the torus, whereas the Alfvén wave propagating toward the southern Jovian hemisphere has a long distance to travel in the torus. Hence, the equatorial lead angle corresponding to the northern spot is small, and the one related to the southern spot is large.

[28] To quantify the longitudinal accuracy of our model, we could fit the position of the footprint of the magnetic field line passing by Io to the position of the most upstream spot observed on Jupiter (TEB or MAW, depending on the longitude), assuming that this spot has a null lead angle. However, as the MAW and the TEB alternate positions, the most upstream spot can have a lead angle of several degrees. It would thus introduce an uncertainty of a few degrees in our mapping. As we show hereafter, the MAW lead angle can be predicted with a smaller uncertainty of $\sim 1^{\circ}$.

[29] The northern and southern lead angles vary depending on the relative latitudes of Io and the plasma torus, with opposite phases. From the Alfvén interaction model and the observations of the latitude of the plasma torus versus its longitude, the northern equatorial lead angle can be modeled as a function of Io's longitude $\lambda_{I o}$ by

$$
\alpha_{N} \simeq A-B \cos \left(\lambda_{I o}-20^{\circ}\right)
$$

The southern equatorial lead angle experiences the opposite variation:

$$
\alpha_{S} \simeq A+B \cos \left(\lambda_{I o}-20^{\circ}\right)
$$

The $A$ and $B$ coefficients are not precisely known. The amplitude of the variation $B$, however, can be estimated from the observation of the UV secondary spot. From the sketch in Figure 1, we can see that the equatorial lead angle 
Table 2. Summary of the Different Characteristics of the Jovian Internal Magnetic Field Models Cited in the Present Paper ${ }^{\mathrm{a}}$

\begin{tabular}{|c|c|c|c|c|c|}
\hline & O6 & VIP4 & VIT4 & Grodent et al. [2008a] & VIPAL \\
\hline Order and degree Uses & third & fourth & fourth & fourth +1 dipole & fifth \\
\hline Magnetic field measurements & yes & yes & only azimuthal component & no & yes (low weight) \\
\hline Io's footpaths & no & yes & yes & yes & yes \\
\hline Io's footprint longitude & no & no & no & no & yes \\
\hline \multicolumn{6}{|l|}{ Matches } \\
\hline Magnetic field measurements & yes & yes & yes & no (not normalized) & above $4 R_{J}$ only \\
\hline Io northern footpath ${ }^{\mathrm{b}}$ & no & roughly & yes & almost perfectly & yes \\
\hline Io southern footpath ${ }^{\text {b }}$ & no & yes & yes & no & yes \\
\hline Europa/Ganymede ${ }^{\mathrm{b}}$ Northern footpath & no & no & no & yes & yes \\
\hline Europa/Ganymede ${ }^{\mathrm{b}}$ Southern footpath & no & yes & yes & no & yes \\
\hline Io's footprint ${ }^{\mathrm{b}}$ longitude & no & no & no & no & yes \\
\hline Maximum radio frequency & no & no & no & no & yes \\
\hline
\end{tabular}

${ }^{\mathrm{a}}$ For satellite footprint latitude $<70^{\circ}$, typical error on longitude and latitude is $1^{\circ}$. For satellite footprint latitude from $70^{\circ}$ to $80^{\circ}$, typical error on longitude is $2^{\circ}-5^{\circ}$ and typical error on latitude is $1^{\circ}-2^{\circ}$. For satellite footprint latitude $>80^{\circ}$, typical error on longitude is $5^{\circ}-10^{\circ}$ and typical error on latitude is $2^{\circ}-5^{\circ}$.

${ }^{\mathrm{b}}$ The model is considered to match the observations if the error on the fit is about or less than $1^{\circ}$.

of the UV spot due to the first reflection of the Alfvén wave is, in the northern hemisphere:

$$
\alpha_{N}(\text { second spot }) \simeq A+B \cos \left(\lambda_{I o}-20^{\circ}\right)+C
$$

And in the southern one:

$$
\alpha_{S}(\text { second spot }) \simeq A-B \cos \left(\lambda_{I o}-20^{\circ}\right)+C
$$

where $\mathrm{C}$ is the delay due to the travel of the wave across the whole torus $(C \simeq A+B)$. The longitude difference between the main spot and the first reflected spot is then $C \pm 2 B$ cos $\left(\lambda_{I o}-20^{\circ}\right)$, depending on the hemisphere. This difference can be measured with high accuracy (typical $1^{\circ}$ error).

[30] The measurements have been performed by Bonfond et al. [2009] and are reported in Figure 1c. They give a value of $B=2.5^{\circ}$ to $4^{\circ}$ and of $C \simeq 2 B$ (which means $A \gtrsim B$ ). In the following we choose to model the equatorial lead angle by $B=3.5^{\circ}$, and the value of $A$ is expected to be close to $3.5^{\circ}-4^{\circ}$ ( $A$ must be larger than $B$ to avoid unphysical negative lead angles).

[31] Figure 4 shows the longitudinal difference between the Io position and the mapping of the field lines connected to Io's UV footprints computed with the VIP4 model (red lines). The variation of the expected equatorial lead angle is shown by the dashed lines. The equatorial lead angle predicted by the VIP4 model deviates from the expected values by up to almost $15^{\circ}$.

\subsection{Magnetic Field Measurements}

[32] The most constraining measurements of the magnetic field close to Jupiter have been performed by the Voyager 1 magnetometer (MAG) [Behannon et al., 1977] and the Pioneer 10 and 11 Helium Vector Magnetometer (HVM) [M. A. Acuna and N. F. Ness, 1975]. These three flybys are the closest ever made to Jupiter. Measurements close to the planet avoid, in part, the effect of the strong current sheet magnetic field, and constrain higher orders of the spherical harmonics decomposition of the internal magnetic field. However, these three flybys cover a very restricted region of the magnetosphere and give only sparse measurements of the magnetic field versus longitude, latitude and distance [Connerney, 1981]. They are thus not constraining enough to determine by inversion a unique magnetic field model of degree/order larger than 3 (the O6 model is truncated at third order of a sixth order fit).

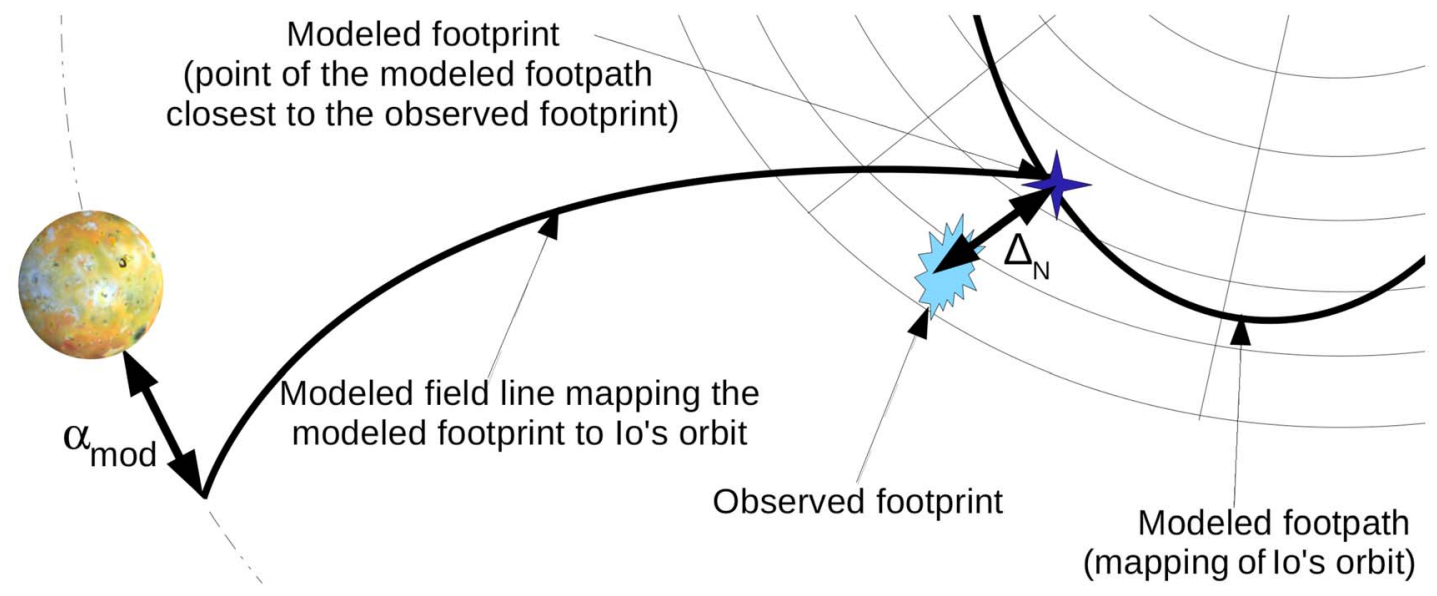

Figure 5. Sketch summarizing the modeling of the Io footpath and of an Io footprint. The modeled Io footprint is the point of the modeled Io footpath closest to the observed Io footprint. Two of the values used to determine the quality of the fit $\left(\Delta_{N}\right.$ and $\left.\alpha_{\text {mod }}\right)$ are shown. 
[33] In the present paper, we use the Voyager and Pioneer measurements as a complement to the observations of the Io footprint positions to determine our model. They mainly provide a way to normalize our coefficients (the topology of a magnetic field does not vary if all the Schmidt coefficients are multiplied by the same value). These measurements also bring more constraints on the free parameters, since a fifthdegree-fifth-order internal magnetic model cannot be entirely constrained by the Io footprint position only (see discussion below). Before using the Voyager and Pioneer measurements to constraint our model, it is necessary to subtract the magnetic field generated by the current sheet. The current sheet magnetic field is computed using the Connerney [1981] model.

[34] In order to estimate the accuracy of the fit of the magnetic field, it is necessary to pay attention to the statistical weight of each measurement: two measurements performed in very different regions are more constraining than two measurements performed at the same location. We organized the measurements by hemispheres and bins of $10^{\circ}$ in longitude and $0.35 R_{J}$ in $\mathrm{L}$ shell. Each measurement weight is the inverse of the number of measurements in its bin. Then the accuracy of the fit of the magnetic field measurements is given by

$$
\Delta B=\sqrt{\frac{\sum_{\text {observations }} \mathcal{W}_{\text {obs }}\left(\frac{\mathbf{B}_{\text {obs }}-\mathbf{B}_{\text {mod }}}{\mathbf{B}_{\text {obs }}}\right)^{2}}{\sum_{\text {observations }} \mathcal{W}_{\text {obs }}}}
$$

where $\mathbf{B}_{o b s}$ is the observed magnetic field vector, $\mathbf{B}_{m o d}$ the modeled one, and $\mathcal{W}_{o b s}$ the statistical weight of each observation. In the present paper, we used only the measurements performed closer than $10 R_{J}$ to Jupiter, i.e., in a region where the current sheet is well described.

\subsection{Surface Field and Radio Emissions}

\subsubsection{Surface Field}

[35] The auroral emissions due to the Io-Jupiter interaction have been observed in the decametric radio range [Burke and Franklin, 1955]. They were related to this interaction by their dependence on the observed phase of Io during the emissions [Bigg, 1964], the Io-related radio emissions being mostly observed when Io is near the limb of Jupiter. Later observations divided the Io emissions in four domains [Boischot et al., 1981; Carr et al., 1983]: The Io-A emissions have a mostly right-handed polarization and correspond to sources in the northern hemisphere when Io has a phase around $230^{\circ}$ (the phase is measured in the positive trigonometrical sense from the antiobserver direction); the Io-B one are right handed and correspond to northern hemisphere sources for Io's phase around $90^{\circ}$; Io-C are left handed and correspond to southern hemisphere sources for Io's phase around $230^{\circ}$; and finally, the lefthanded Io-D emissions correspond to southern sources for Io's phase around $90^{\circ}$.

[36] Figure 6 shows a typical Io-B dynamic spectrum. All the Io-related emissions appear arc shaped, with an intense quasi-vertical branch in this plot (that is with a large frequency drift rate) and a weaker branch with a lowerfrequency drift rate. According to Queinnec and Zarka [1998] and Hess et al. [2008], the faint slowly drifting branch of the Io-B arcs should correspond to emissions very close to the surface frequency.

[37] The radio emissions in the auroral regions are mainly caused by the cyclotron maser instability (CMI) (see reviews by Zarka [1998] and Treumann [2006]). This instability involves the resonance between a circularly polarized wave and the gyration motion of the electrons around the magnetic field lines (see reviews by $W u$ [1985] and Treumann [2006]). The emission occurs at the local electron cyclotron frequency $f_{c e}=\left|\frac{q_{e} B}{2 \pi m_{e}}\right|$ (with $q_{e}$ the electron electric charge, $m_{e}$ the electron mass and $B$ the local magnetic field strength) between $\sim 3 \mathrm{MHz}$ and $\sim 40 \mathrm{MHz}$ [Queinnec and Zarka, 1998; Zarka et al., 2001]. This linear relation between emission frequency and magnetic field strength permits one to obtain the amplitude of the magnetic field in the radio sources from the radio observations. Theoretically, the radio emissions could provide measurements of the magnetic field at high latitude and low altitude. However, the long wavelengths of the radio emissions imply a very low angular resolution in the observations, so that we cannot pinpoint accurately the position of the radio source [Zarka, 2004].

[38] Even without angular resolution, important data can be extracted from the radio observations. For a given longitude of Io, the maximum emitted frequency cannot be larger than the electron cyclotron frequency at the top of the Jovian ionosphere. Hence, the radio emissions give minimum values of the magnetic field strength at the surface of the planet. The first measurements of the maximum radio frequency versus Io's longitude has been performed by Genova and Aubier [1985]. These measurements, for the northern and southern hemisphere sources, respectively, are shown as green circles in Figures $7 \mathrm{a}$ and $7 \mathrm{~b}$. The electron cyclotron frequency deduced from the VIP4 model, at the location of the UV footprints of Io and as a function of Io's longitude, is shown on top of the measurements. In the northern hemisphere, the observed maximum frequencies clearly exceed the surface electron cyclotron frequency predicted by the model for Io's longitudes between $\sim 180^{\circ}$ and $\sim 280^{\circ}$. This excess reaches more than $10 \mathrm{MHz}$ near a longitude of $250^{\circ}$. This suggests that the VIP4 model does not give the correct surface magnetic field for these longitudes in the northern hemisphere.

[39] Further measurements were performed [Zarka et al., 2002], separating the contributions of the Io-A and Io-B arcs. These measurements are represented in Figure 7 as the blue (Io-A) and red (Io-B) dots. These measurements confirm those of Genova and Aubier [1985] and bring more details about the emissions of interest. Most of the emissions occurring at frequencies above the surface electron cyclotron frequency deduced from the VIP4 model correspond to Io-B emissions. A more careful examination of these emissions shows that they correspond to the faint, slowly drifting branch of the Io-B arcs. These emissions should occur very close to the surface electron cyclotron frequency [Hess et al., 2008], thus giving an approximate value of the magnetic field strength on the top of the Jovian ionosphere in this region. These measurements show that the surface magnetic field strength given by the VIP4 model does not match the surface magnetic field strength at Jupiter in this region. 
a) Io-B observation
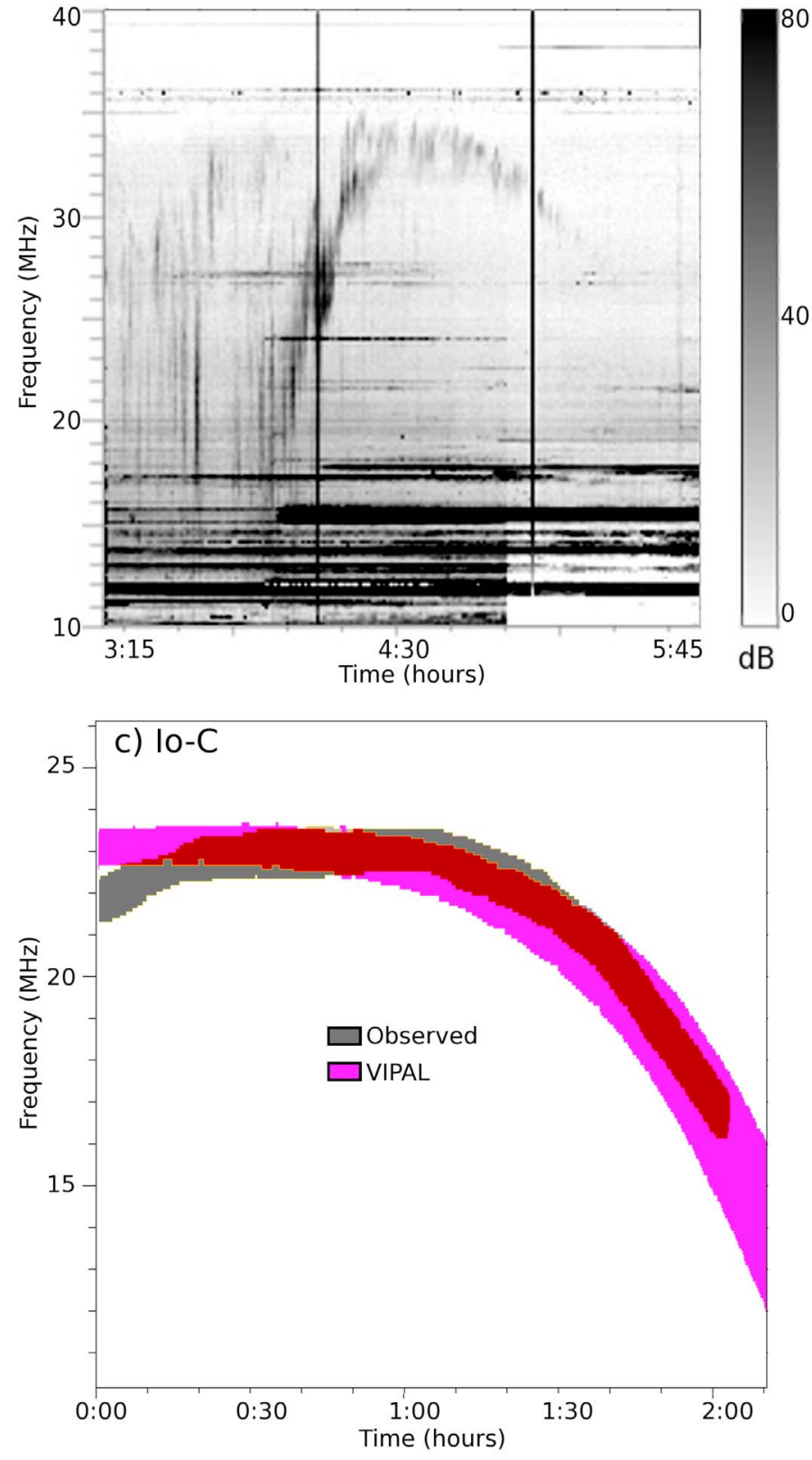

b) lo-B
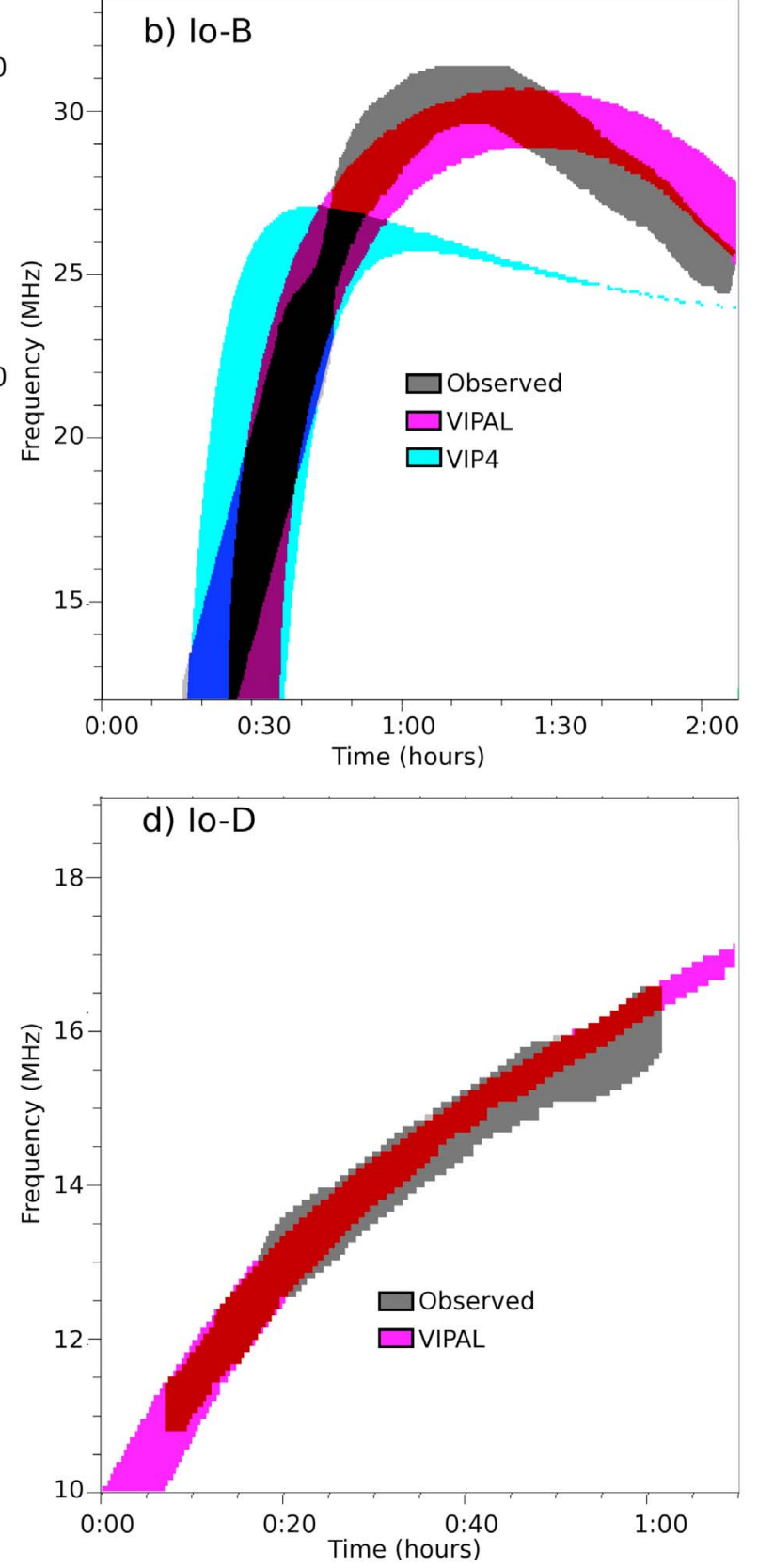

Figure 6. Modeling of radio arcs observed at the Nançay decameter array by the ExPRES code, using the VIPAL and VIP4 magnetic field models. (a) Dynamic spectrum of a typical Io-B radio arc recorded at Nançay on 16 July 2009. (b) Modeling of the dynamic spectrum of an Io-B arc, recorded on 3 April 2008. The fits of this arc by using the VIPAL and VIP4 models are superimposed. The VIP4 model cannot be used to fit the Io-B arcs since it does not allow high frequency to be emitted above the Jovian surface. (c) Modeling of the dynamic spectrum of an Io-C arc, recorded on 20 October 1998. The fit of this arc by using the VIPAL model is superimposed. (d) Modeling of the dynamic spectrum of an Io-D arc, recorded on 27 May 1996. The fit of this arc by using the VIPAL model is superimposed.

\subsubsection{Magnetic Field Line Topology}

[40] The large uncertainties in the northern hemisphere surface field has also been noted by Hess et al. [2010a], who tried to reproduce the shape of the radio emissions in the time-frequency plane by simulating the geometry of the observations and computing the visibility of the sources. The shape of the Io-controlled radio emissions has been explained by Hess et al. [2008] and Ray and Hess [2008] as being due to the highly anisotropic and frequency-dependent beaming pattern of the radio sources. The Io-related emissions are continuously emitted at all possible frequencies along the emitting magnetic field lines, but they can only be observed when the observer is in the direction of emission, which varies with the frequency. The arc shape of the 

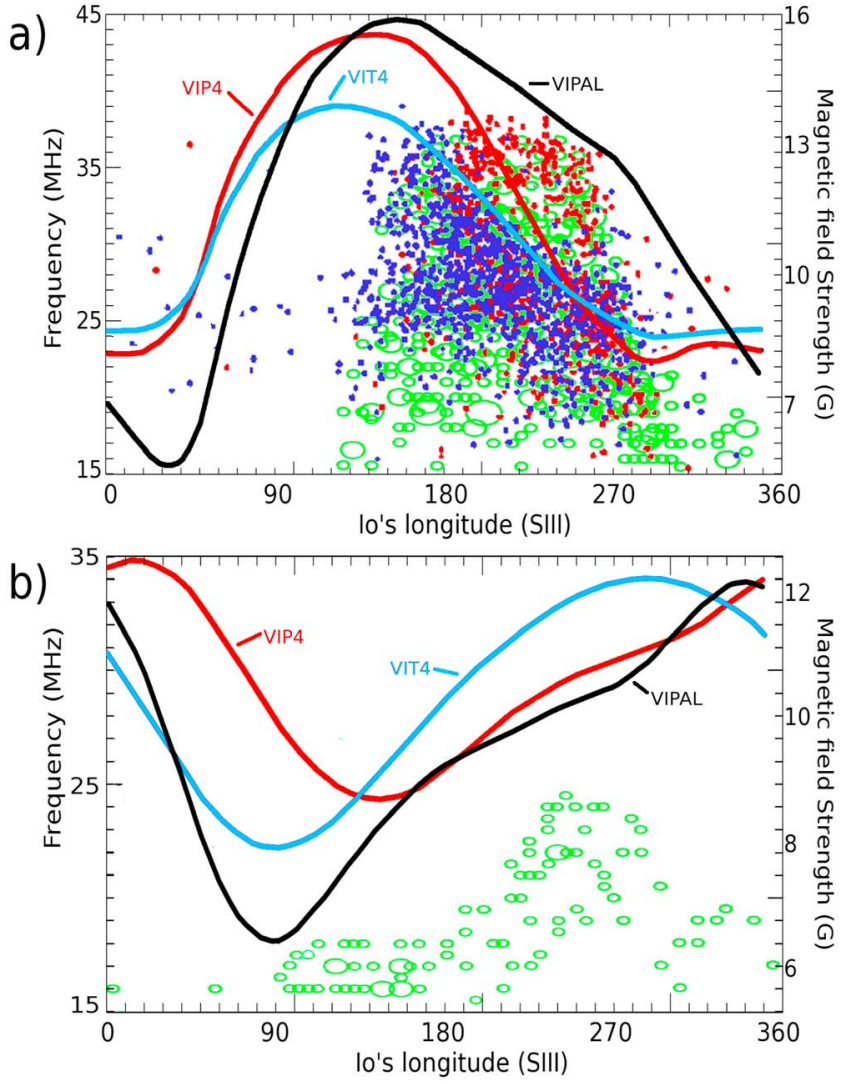

Figure 7. Maximum radio frequencies observed versus Io's longitude for (a) the Jovian northern hemisphere and (b) the Jovian southern hemisphere. The frequencies observed by Genova and Aubier [1985] are shown by the green circles, whereas blue and red dots correspond to the Io-A and Io-B maximum frequencies, respectively, as observed by Zarka et al. [2002]. Lines stand for the surface electron cyclotron frequencies along Io's footpath: black lines stand for the VIPAL model, red lines stand for the VIP4 model, and blue lines stand for the VIT4 model.

emissions in the time frequency plane results from this visibility effect.

[41] Hess et al. [2008] developed the ExPRES (Exoplanetary and Planetary Radio Emissions Simulator) numerical code to compute the visibility of the sources, taking into account the geometry of the observation and the anisotropy of the emissions. This code models the radio arcs, assuming that the sources are distributed along a magnetic field line corotating with Io, i.e., with a constant equatorial lead angle. The dynamic spectrum of the emissions depends only on the lead angle of the emitting field line and on the energy of the emitting particles, which controls the direction of emission.

[42] Hess et al. [2010a] fitted the observed arcs with simulated ones by varying both the electron energy and the lead angle. They used the VIP4 model to describe the topology of the magnetic field lines. They successfully reproduced the southern arcs, but pointed out that the model could be improved by assuming a field line corotating with the UV footprints instead of one corotating with Io. This suggests that the azimuthal description of the magnetic field lines by the VIP4 model was not accurate enough. Moreover they were unsuccessful at modeling the northern arcs, since the VIP4 model cannot account for the observed frequencies.

[43] The quality of the radio arc fit depends both on the accuracy of the hypothesis (constant velocity, negligible refraction, ...) and on the accuracy of the description of the magnetic field line topology by the magnetic field model. It thus constitutes an important test to determine the validity of a new magnetic field model.

\section{Magnetic Field Determination}

[44] In this section we present the method we used for determining a magnetic field model based on a spherical harmonic decomposition, with the goal of improving the mapping of the Io footprints relative to the VIP4 magnetic model. We proceeded in three steps. Since the longitudinal position of the Io footprints is the value which is the less accurately described by the VIP4 model, we first modified the VIP4 coefficients to correct this longitudinal error.

[45] However, this modification of the coefficients of the spherical harmonics leads to a different mapping of the Io footpath, which is not as accurate as the one given by the VIP4 model. Thus, in a second step, we modify the spherical harmonics coefficients to fit both the longitude and also the latitude of the Io footprints, and the magnetic field measurements of made by the Voyager 1 and Pioneer 10 and 11 spacecraft.

[46] We then obtained a model which provides a better mapping of the Io footprint location and is consistent with the magnetic field measurements. However, this model is not unique and other sets of coefficients would provide an equally good model. We selected the solution comprising coefficients with the smallest amplitude needed for an accurate fit. That is, we minimized the coefficients while keeping the accuracy on the fit of the Io footprints close to that obtained in the second step.

\subsection{Longitudinal Correction}

[47] The equatorial longitude of the magnetic field line mapping to Io's UV footprints $\left(\lambda_{m f l}\right)$ can be written as the longitude of Io $\left(\lambda_{I o}\right)$ minus a lead angle $\left(\alpha_{N / S}\right)$ :

$$
\lambda_{m f l}=\lambda_{I o}-\alpha_{N / S}
$$

As shown in section 3.3, the lead angles vary with Io's longitude as

$$
\alpha_{N / S} \simeq A \pm 3.5 \cos \left(\lambda_{I o}-20^{\circ}\right)
$$

where the sign in front of the cosine depends on the hemisphere (negative sign for the northern, positive sign for the southern), and $A \simeq 4^{\circ}$. In the mean time, an equatorial lead angle can be obtained from the magnetic field model. It corresponds to the difference between the longitude of Io and the equatorial longitude of a modeled field line, whose ionospheric foot matches the observed UV footprint.

$$
\alpha_{\text {mod }}=\lambda_{I o}-\lambda_{m f l}
$$

However, as the Io footpaths are not perfectly modeled, a magnetic field line connected to the observed location of the UV footprint may not cross the Io orbit. Hence, we first 
compute the footpaths corresponding to the Io orbit, and choose the point of the footpath closest to the observed UV footprint as the footprint of the modeled field line.

[48] The goal of our modeling is to obtain a modeled lead angle, such as

$$
\alpha_{\text {mod }}=A^{\prime} \pm 3.5 \cos \left(\lambda_{I o}-20^{\circ}\right)
$$

To do so, we first perform a Fourier transform of the difference between modeled and observed lead angles $\left(\delta \alpha_{N / S}=\right.$ $\left.\alpha_{m o d}-\alpha_{N / S}\right)$, for each hemisphere independently:

$$
\begin{aligned}
\delta \alpha_{N} & =\sum_{m} C_{N m} \cos (m \phi)+D_{N m} \sin (m \phi) \\
\delta \alpha_{S} & =\sum_{m} C_{S m} \cos (m \phi)+D_{S m} \sin (m \phi)
\end{aligned}
$$

The lead angle differences involve only azimuthal variation and the magnetic field lines follow to a first approximation the gradient of the potential field. Hence, we minimize the Fourier components of the lead angle differences using spherical harmonics with the same azimuthal periodicity. For each spherical harmonic orders $m$, we chose to limit ourselves to the spherical harmonics with the minimum variation with latitude, i.e., with the degrees $m$ and $m+1$. Hence, our magnetic field model uses harmonics up to the fifth order, i.e., one order more than the VIP4 model. The following equations give the correction applied to the Schmidt coefficients to minimize the Fourier components of order $m$ of the lead angle differences $\left(D_{N_{m}}, D_{S_{m}}, C_{N_{m}}\right.$ and $\left.C_{S_{m}}\right)$ :

$$
\begin{aligned}
& g_{m}^{m}=g_{m}^{m}+\epsilon\left(D_{N m}-D_{S_{m}}\right) \\
& g_{m+1}^{m}=g_{m+1}^{m}+\epsilon\left(D_{N m}+D_{S_{m}}\right) \\
& h_{m}^{m}=h_{m}^{m}-\epsilon\left(C_{N m}-C_{S m}\right) \\
& h_{m+1}^{m}=h_{m+1}^{m}-\epsilon\left(C_{N m}+C_{S m}\right)
\end{aligned}
$$

The $\epsilon$ term is set to a small value $(\ll 1)$ to ensure convergence, in the present computations we choose $\epsilon=0.01$. Preliminary computations showed that the lead angle difference terms are important up to the $m=4$ order, so we limited our correction to this order. However, as showed hereafter, the correction of the $m=4$ terms in the lead angle difference involves spherical harmonics up to the $m=5$ order. The correction is applied by iteration. At each step, we compute the mean lead angle difference for each hemisphere:

$$
\Delta \alpha_{N / S}=\sqrt{\frac{\sum_{\text {observations }} \delta \alpha_{N / S}^{2}}{N_{\text {observations }}}}
$$

We iterated until the lead angle differences $\Delta \alpha_{N / S}$ reach their minimum values. Between each iteration, the Io footpaths are recomputed, so the positions of the footprints of modeled magnetic field lines are modified to ensure that the magnetic field lines always cross the Io orbit. Since there is no magnetic monopole, $g_{0}^{0}=0$. Thus the $m=0$ component of the lead angle difference cannot be modified. This implies that we cannot force the $A$ and $A^{\prime}$ coefficients of equations (10) and (12) to be equal. The modeled lead angles may thus suffer from a constant difference with the observed ones.
[49] The minimization is obtained in a few tens of iterations. The lead angles in the southern hemispheres fit equation (12) with a mean error of $0.3^{\circ}$ and the northern one with a mean error of $1.2^{\circ}$. However, the Io footpaths obtained from our model do not satisfactorily fit the observed ones: the mean angular distance between the observed footprint positions and the closest point on the modeled footpaths is $2^{\circ}$ in the northern hemisphere (versus $1.5^{\circ}$ in for the VIP4 model) and $1.3^{\circ}$ in the southern one (versus $0.9^{\circ}$ for the VIP4 model). Moreover, in the northern hemisphere this difference is concentrated in a sector of longitude corresponding to the location of the Grodent et al. [2008a] anomaly (between $70^{\circ}$ and $140^{\circ}$ ). In this sector the modeled footpath does not reproduce at all the observed one. Hence, after fitting the equatorial lead angles, we also fitted the modeled Io footpaths to the observed ones.

\subsection{Latitudinal Correction}

[50] The magnetic field models are usually computed by using a residual minimization method, generally a gradient method, to invert the magnetic field measurements. This type of method consists in computing at each step, how the error on the fit of each measurement varies when each input parameter is changed independently. Then, the solution is obtained by searching which linear combination of parameter perturbations produces the smallest error. This type of method can be applied if (1) the errors on the fit to the measurements vary linearly with the parameter change and (2) the variation of the error on the fit due to the change of a given parameter does not depend on the way the other parameters change (at least if the variation is small). In other terms, the problem has to be linear for small variations: if we note $y_{i}$ the $i$ th measurements and $x_{j}$ the $j$ th input parameter, the variation of $y_{i}$ can be written:

$$
\Delta y_{i}=\sum_{j} \frac{\partial y_{i}}{\partial x_{j}} \delta x_{j}+\sum_{k} \frac{\partial^{2} y_{i}}{\partial x_{j} \partial x_{k}} \delta x_{j} \delta x_{k}+\ldots
$$

The gradient residual minimization methods can be applied when all but the terms of order 1 can be neglected. By choosing a small enough $\delta x_{j}$, it is always possible to neglect the second-orders terms. The fit of the magnetic field measurements, used to determine most of the internal magnetic field models, is a linear problem, and the above method can be used. In order to compute the VIP4 model, Connerney et al. [1998] also fitted the position of the Io footpath. This fit is nonlinear, but its weight in the magnetic field determination was smaller than that of the magnetic field measurements, which permitted the convergence of the gradient method.

[51] In the present study, the fit of the position of the Io footprints has a far larger weight than that of the magnetic field measurements (see below). This problem is highly nonlinear, since the position of the magnetic line footprints strongly depends on the potential field gradients along the whole magnetic field line. In this case, the variations of the footprint locations induced by a change of a given Schmidt coefficient are strongly dependent of the variations induced by the change of the other Schmidt coefficients. The numerical computation of a few ratios between secondorder and first-order terms $\left(\frac{\partial y_{i}}{\partial x_{j}}\right)\left(\frac{\partial^{2} y_{i}}{\partial x_{j} \partial x_{k}}\right)^{-1}$ showed that for 
coefficients of degree/order larger than 2-3 the maximum value of $\delta x_{j}$ on which the problem can be considered linear is very small $(<0.0001)$. Still, the VIT4 internal magnetic field model [Connerney, 2007], which most depends on the fit of the Io footpaths, was determined by using a gradient method with gradients computed numerically. However, this model is limited to the fourth order/degree and does not fit the longitudinal position of the spots (which complicate the problem). This may explain why Connerney [2007] succeeded in converging using larger steps.

[52] In our study, the use of any gradient method would then take a very long time. Hence, the linear residual minimization methods are not able to produce a magnetic field model by inverting the position of the Io footprints.

[53] To fit the Io footprint positions, we proceed by successive trials: we successively add or subtract a small value to a Schmidt coefficient and we test the quality of the fit. If the fit is better, we definitively add or subtract this value. We started with the Schmidt coefficients obtained from the lead angle fit. We proceed Schmidt coefficient after Schmidt coefficient, beginning with those corresponding to the lowest $n$ and $m$ orders. We begin with a variation of \pm 0.04 and then $\pm 0.02, \pm 0.01, \pm 0.005, \pm 0.0025, \pm 0.00125$, \pm 0.000625 and \pm 0.00031 . The whole process has been iterated until the values of the Schmidt coefficients stabilized. This method permits us to overcome the strong dependency between the Schmidt coefficients, as we do not change multiple Schmidt coefficients at the same time. The tendency of gradient minimization methods to converge toward local minima is due to the fact that the amplitude of the change in Schmidt coefficient decreases at each iteration (it is a necessary condition for the convergence). In our method, this amplitude decreases (from 0.04 to 0.00031), but then we start again beginning again by an amplitude of 0.04 . This permits us to avoid some local minima, although we cannot guarantee that the final solution is that with the global minimum.

[54] To test the quality of the fit we used five indicators: The mean angular distance between the observed Io footprints and the closest point of the modeled footpaths in both hemispheres $\left(\Delta_{N / S}\right.$, equation (3)), the difference between the observed and modeled equatorial lead angles in both hemispheres $\left(\Delta \alpha_{N / S}\right.$, equation (15)), and the mean error on the fit of the Voyager 1 and Pioneer 10 and 11 magnetic field measurements ( $\Delta B$, equation (8)). The quantity we minimize is

$$
\mathcal{D}=\frac{\Delta_{N}}{1^{\circ}}+\frac{\Delta_{S}}{1^{\circ}}+\frac{\Delta \alpha_{N}}{1^{\circ}}+\frac{\Delta \alpha_{S}}{1^{\circ}}+\frac{\Delta B}{\Delta B_{\mathrm{VIP} 4}}
$$

where $\Delta B_{\mathrm{VIP} 4}$ is the error on the fit of the magnetic field measurements for the VIP4 magnetic model. Each indicator is normalized by the value we expect to reach (e.g., the angular distances are expected to be smaller than $1^{\circ}$ at the end of the fit).

[55] The method converges toward a solution in a few tens of iterations. The result is a spherical harmonic model of Jupiter's internal magnetic field which permits a far better mapping of the Io footprints. However, this model is not unique (see Discussion), similar to all the Jovian internal magnetic field models previously computed.

\subsection{Schmidt Coefficient Minimization}

[56] The minimization method developed by Connerney [1981], and used for the O6 and VIP4 models, not only minimizes the error to the fit of the measurements, but also minimizes the spherical harmonics coefficients. In the same way, from all the possible fifth-order and fifth-degree magnetic field models, we arbitrarily select one with the smallest Schmidt coefficients as possible. To do so, we simply reuse the above method, but instead of minimizing the $\mathcal{D}$ value, we minimize a function which associate the accuracy of the fit and the power in the nondipolar Schmidt coefficients:

$$
\begin{gathered}
\mathcal{D}^{\prime}=\tan \left(\pi \frac{\mathcal{D}-\mathcal{D}_{0}}{\mathcal{D}_{0}}\right)+\frac{\mathcal{S}}{\mathcal{S}_{0}} \\
\mathcal{S}=\sum_{n=2}^{5} \sum_{m=0}^{n} \sqrt{\left(g_{n}^{m}\right)^{2}+\left(h_{n}^{m}\right)^{2}}
\end{gathered}
$$

where $\mathcal{D}_{0}$ is the value of $\mathcal{D}$ obtained from the previous step, i.e., the best fit without any constraint on the Schmidt coefficients. $\mathcal{S}_{0}$ is the sum of the nondipolar Schmidt coefficients before we minimize them. The expression of $\mathcal{D}$ is chosen to limit the variation of the error on the fit measured by $\mathcal{D}$; that is, we want the final solution to fit the observation almost as well as the solution without the Schmidt coefficient minimization. The tan function permits us to allow for small variations of the fit accuracy $\mathcal{D}$, but forbids large ones. The expression we chose limits the variation of the error on the fit $\mathcal{D}$ to $25 \%$ at most, if all the nondipolar terms become equal to 0 (if $\mathcal{S} \rightarrow 0$ ).

[57] The minimization of the Schmidt coefficients actually increases the error on the fit by $3 \%$, whereas it decreases the sum of the amplitude of the nondipolar Schmidt coefficients by more than $15 \%$. This $3 \%$ increase of the error on the fit is small. As a comparison, the error on the fit using VIP4 is $\sim 300 \%$ larger than the error on the fit using VIPAL (mostly because of the error on the azimuthal component of the magnetic field).

\section{Magnetic Field Coefficients}

[58] Figure 2 shows the surface fields in the northern and southern hemispheres for the VIP4, Grodent et al. [2008a] (northern hemisphere only), and VIPAL models. Compared to the VIP4 model, the northern hemisphere magnetic field flux in our model reaches higher value and draws an horseshoe pattern, centered on the north Jovigraphic pole between the latitudes of $\sim 30^{\circ}$ to $\sim 70^{\circ}$. This pattern is also seen for the Grodent et al. [2008a] model. It is shown in section 6.3 that this pattern permits a better description of the maximum frequencies of the radio emission along the northern Io footpath.

[59] The Schmidt coefficients obtained by the above method are listed in Table 3. Table 3 also shows the VIP4 and VIT4 Schmidt coefficients [Connerney et al., 1998] for comparison. The comparison of high-degree/order terms is far less significant than the comparison of the low-degree/ order ones, since a small modification of low-degree/order terms introduces a large variation of the high orders, whatever the method and the data used to determine them. 
Table 3. VIP4, VIT4, and VIPAL Schmidt Coefficients ${ }^{\mathrm{a}}$

\begin{tabular}{|c|c|c|c|c|}
\hline Coefficient & VIP4 & VIT4 & VIPAL & $\delta_{5 \%}$ \\
\hline$g_{1}^{0}$ & 4.205 & 4.281 & 4.2 & \\
\hline $\mathbf{g}_{2}^{\mathbf{0}}$ & -0.0512 & -0.0428 & 0.6441 & 0.005 \\
\hline$g_{3}^{0}$ & -0.0158 & -0.0891 & -0.1058 & 0.01 \\
\hline $\mathbf{g}_{4}^{0}$ & -0.1676 & -0.2293 & -0.7466 & 0.01 \\
\hline$g_{5}^{0}$ & & & -0.066 & 0.02 \\
\hline $\mathbf{g}_{l}^{l}$ & -0.6592 & -0.7531 & -0.6975 & 0.002 \\
\hline$g_{2}^{1}$ & -0.619 & -0.5943 & -0.8672 & 0.004 \\
\hline$g_{3}^{1}$ & -0.5204 & -0.2145 & -0.59 & 0.01 \\
\hline$g_{4}^{1}$ & 0.2221 & 0.1894 & 0.3282 & 0.01 \\
\hline$g_{5}^{1}$ & & & 0.0737 & 0.01 \\
\hline $\mathbf{g}_{2}^{2}$ & 0.4969 & 0.4439 & 0.9598 & 0.005 \\
\hline$g_{3}^{2}$ & 0.244 & 0.2113 & 0.6322 & 0.01 \\
\hline $\mathrm{g}_{4}^{2}$ & $-\mathbf{0 . 0 6 0 7}$ & -0.0385 & -0.338 & 0.01 \\
\hline$g_{5}^{2}$ & & & -0.1711 & 0.01 \\
\hline$g_{3}^{3}$ & -0.176 & -0.0119 & 0.4671 & 0.02 \\
\hline$g_{4}^{3}$ & -0.2024 & 0.0993 & 0.1826 & 0.02 \\
\hline$g_{5}^{3}$ & & & -0.1793 & 0.02 \\
\hline$g_{4}^{4}$ & 0.0664 & 0.0127 & -0.1429 & 0.02 \\
\hline$g_{5}^{4}$ & & & -0.0077 & 0.02 \\
\hline$g_{5}^{5}$ & & & -0.0740 & 0.04 \\
\hline$h_{1}^{1}$ & 0.25 & 0.2462 & 0.1973 & 0.002 \\
\hline$h_{2}^{1}$ & -0.3605 & -0.5015 & -0.4041 & 0.004 \\
\hline$h_{3}^{1}$ & -0.088 & -0.1719 & -0.231 & 0.01 \\
\hline$h_{4}^{1}$ & 0.0756 & 0.1609 & 0.3283 & 0.01 \\
\hline$h_{5}^{1}$ & & & 0.2065 & 0.01 \\
\hline $\mathbf{h}_{2}^{2}$ & 0.0525 & 0.3845 & 0.603 & 0.005 \\
\hline$h_{3}^{2}$ & 0.4083 & 0.4067 & 0.516 & 0.01 \\
\hline $\mathbf{h}_{4}^{2}$ & 0.4041 & 0.1181 & -0.2131 & 0.01 \\
\hline$h_{5}^{2}$ & & & -0.1167 & 0.01 \\
\hline$h_{3}^{3}$ & -0.3159 & -0.3526 & -0.1131 & 0.02 \\
\hline$h_{4}^{3}$ & -0.166 & 0.062 & -0.0606 & 0.02 \\
\hline$h_{5}^{3}$ & & & -0.0288 & 0.02 \\
\hline$h_{4}^{4}$ & 0.0387 & 0.0126 & -0.0486 & 0.02 \\
\hline$h_{5}^{4}$ & & & -0.0050 & .02 \\
\hline$h_{5}^{5}$ & & & -0.2279 & 0.04 \\
\hline
\end{tabular}

${ }^{a}$ The most important differences are indicated in bold and are discussed in the text. The $\delta_{5 \%}$ value shows the sensitivity of our fit depending on the coefficient degree and order. It corresponds to the average variation to apply to the coefficients of a given degree and order to induce a $5 \%$ variation of the $\mathcal{D}$ fitting coefficient. Only the fifth-degree-fourth-order terms are smaller than this sensitivity measure. This measure accounts for the sensitivity of each coefficient independently and does not correspond to the uncertainty on the coefficients. Italic font indicates the coefficients whose value is lower than $\delta_{5 \%}$.

[60] The magnetic dipole terms $\left(g_{1}^{0}, g_{1}^{1}\right.$ and $\left.h_{1}^{1}\right)$ are essentially the same in the two models. The difference in these terms corresponds to a change of the Jovian magnetic field tilt. In our model this tilt is $\sim 9.8^{\circ}$. This value of the tilt is consistent with the measurements of Russell et al. [2001a]. Our model also gives a shift of $\sim 5^{\circ}$ of the longitudinal direction of the magnetic dipole compared to the one given by the VIP4 model. This shift, which was also present in the VIT4 model, may be due to an error on the estimate of the rotation period of Jupiter. Higgins et al. [1996] proposed, based on 35 years of radio observations of Jupiter, that the rotation rate of the planet interior may be $\sim 25 \mathrm{~ms}$ shorter than the System III(1965) rotation rate and has been adopted by the IAU. This period has since be determined more precisely by Higgins et al. [1997] with more data. The new Higgins et al. [1996] period is at the basis of the ephemeris used to determine the position of the UV footprints. The difference in the rotation rate, between the System III(1965) period and the new IAU period can largely account for the longitude shift, since more than 25 years separate the Voyager magnetic field measurements and the Bonfond et al. [2009] UV observations. Our model suggests that the Jupiter period is closer to the System III(1965) period than to the new period, in agreement with the observations of Russell et al. [2001b].

[61] The major differences between the VIPAL model and the VIP4 and VIT4 models come from the quadrupolar (second-degree) and fourth-degree terms (shown in bold in Table 3). These terms are responsible for the horseshoe shape of the surface magnetic field strength in the northern hemisphere. Since this shape is also present in the Grodent et al. [2008a] model, they can be understood as describing the lowest orders of the magnetic anomaly. The $g_{2}^{0}$ and $g_{4}^{0}$ terms are responsible for the belt of high magnetic strength near a latitude of $40^{\circ}$ north, which is needed for the fit of the Io footpath, particularly between the longitudes of $120^{\circ}$ and $240^{\circ}$. For a fifth-degree internal magnetic model, the only combination of Schmidt coefficients generating such a pattern is $\left(g_{2}^{0}-g_{4}^{0}\right)$. This explains why the sum of Schmidt coefficient $\left(g_{2}^{0}+g_{4}^{0}\right)$ is roughly the same for the VIP4 and VIPAL models, whereas the individual values of $g_{2}^{0}$ and $g_{4}^{0}$ are very different between the models: the mapping of the Io footprint does not depend on this sum, in a first-order approximation, so that the largest constraints on it comes from the magnetic field measurements. Nevertheless, a higher-order model would possibly describes this feature with higher orders. The same is also true for the combinations of the $g_{2}^{2}, h_{2}^{2}$ and $g_{4}^{2}, h_{4}^{2}$ terms, which describe the weakening of the surface magnetic field in the northern hemisphere, for longitudes from $\sim 330^{\circ}$ to $\sim 80^{\circ}$. The weakening is visible in Figure $2 \mathrm{~d}$, as it is responsible for the horseshoe pattern of the magnetic field strength in the northern hemisphere. As for the axisymmetric terms, this pattern can only be obtained by a combination of the $g_{2}^{2}$, $h_{2}^{2}$ and $g_{4}^{2}, h_{4}^{2}$ terms, although it probably involves higher orders.

\section{Comparison With Observations}

\subsection{Io's Auroral Footprints}

[62] Figures $2 \mathrm{~d}$ and 2e, show the position of the model Io footpaths obtained with VIPAL, in the northern and southern hemispheres, respectively. In both cases the footpaths better fit the observed positions of the Io footprints than the VIP4 model. The mean angular distance between the observed and modeled footprints is $0.9^{\circ}$ in the northern hemisphere versus $1.5^{\circ}$ for the VIP4 model, and $0.9^{\circ}$ in the southern hemisphere comparable with the VIP4 model. Nevertheless, the position of the northern footpath does not fit well the position of the observed footprints in a sector of longitude comprised between $\sim 100^{\circ}$ and $\sim 140^{\circ}$. These longitudes correspond to the location of the magnetic anomaly modeled by Grodent et al. [2008a]. The magnetic anomaly is discussed in more details in section 6.2. 
[63] Figure 4 shows the equatorial lead angles from northern and southern hemispheres. The longitudinal variation of the lead angle (equation (10)) is well reproduced. However, the mean value of the lead angle $\left(A^{\prime}\right)$, which is assumed to be close to $A \sim 4^{\circ}$ in both hemispheres, is actually different in the two hemispheres: $A^{\prime}=2.8^{\circ}$ in the northern hemisphere and $A^{\prime}=4.3^{\circ}$ in the southern one. The difference is mainly due to the fact that our lead angle fitting method does not permit to fit the mean lead angle value. The southern mean lead angle value is close to the assumed value, the northern one, however, leads to negative values of the lead angle for some longitudes, when it should always be positive. Nevertheless, our model gives lead angles far closer to their expected values than the VIP4 model; that is, higher orders/degrees would be needed to model such a small-scale structure.

\subsection{Europa and Ganymede Auroral Footprints}

[64] Figure 3 shows the positions of the observed footprints of Io, Europa and Ganymede in the northern (Figure 3a) and southern (Figure 3b) hemispheres (from Table 1), as well as their footpaths modeled with the VIP4 (dashed lines) and VIPAL (solid lines) internal magnetic field models. The magnetospheric component of the magnetic field, generated by the current sheet, was modeled using the axisymmetric Connerney [1981] model.

[65] In the southern hemisphere, the mapping of the Io footpath by the VIP4 and the VIPAL gives a similar mean error $\left(\Delta_{S}\right)$. The southern footpaths of Europa and Ganymede are slightly less well described by our model than by the VIP4 one. However, the mean error on the mapping of the footprints positions is consistent with the uncertainties of the observations. In the northern hemisphere, the Io footpath is slightly better described by the VIPAL model than by the VIP4 one (mean error of $0.9^{\circ}$ versus $1.5^{\circ}$ ). For the Europa and Ganymede ones, however, the difference is far more important. These footpaths are not reproduced by the VIP4 model, whereas the VIPAL model describes the partially observed footpaths from Europa and Ganymede with a mean angular error of a fraction of a degree, except in the anomaly region. Hence, the VIPAL model, although improving the satellite footprint mapping relative to the VIP4 model is not as precise as the Grodent et al. [2008a] model in the northern hemisphere. Nonetheless, the VIPAL model is accurate in the southern hemisphere were the Grodent model is not appliable.

[66] The anomaly region can be defined from Figure 3 as the region where the modeled Ganymede and Io footpaths diverge from the observed location of the footprints, i.e., between $\sim 57^{\circ}$ and $\sim 80^{\circ}$ in latitude and between $\sim 100^{\circ}$ and $\sim 140^{\circ}$ in longitude. The Io and Ganymede footpaths diverge in opposite longitudinal directions, whereas the Europa footpath is well described. This suggests that the Europa footprint actually passes near the center of the magnetic anomaly. This center is around a latitude of $65^{\circ}-70^{\circ}$ north and a longitude of $125^{\circ}-130^{\circ}$, which is close to the value from Grodent et al. [2008a]. The Grodent et al. [2008a] model succeeded in reproducing the kink of the Io and Ganymede footpaths by inserting an additional ad hoc dipole below the planetary surface. Our approach is different and since our model is limited to the fifth-degree-fifth-order spherical harmonics, it cannot describe accurately an anomaly with an extent of less than $40^{\circ}$ in longitude and latitude.

\subsection{Surface Field and Radio Emissions}

[67] Figure 7 shows the maximum frequencies reached by the Io-related radio emissions versus the Io longitude. The maximum frequency versus Io longitude deduced from the VIP4 model is indicated. The VIP4 model cannot explain the maximum frequencies of the emissions in a range of longitudes comprised between $180^{\circ}$ and $270^{\circ}$, since the observed frequency are above the modeled surface electron cyclotron frequency, by as much as $10 \mathrm{MHz}$. The surface electron cyclotron frequency deduced from the VIPAL model match closely the maximum observed frequencies in this longitude range, where most of the emissions are observed.

[68] Figure 6 shows the modeling of the visibility of the radio sources for three different observations. Those are performed using the ExPRES simulation code [Hess et al., 2008]. This code allows to compute the visibility of the radio sources, given an observation geometry (position of the observer, of Io, and Jovian longitude of the observer), a model of the magnetic field line topology, and the velocity of the emitting electrons, which determines the direction of emission. The two unknown parameters in the simulation are the equatorial lead angle of the emitting field line relative to Io and the velocity of the electrons. These parameters can be constrained by fitting the observed radio arcs. Such fits have been performed by Hess et al. [2010a] over more than 60 radio arcs, using the VIP4 model. The authors modeled only southern arcs, as their attempts to model northern ones showed that the surface magnetic field of the VIP4 model was to low to allow emissions at the frequencies observed. The authors concluded from the measurements that the VIP4 model was not accurate enough to allow for a precise determination of the lead angle. However, the variation of the lead angle with Io's longitude suggested that the radio arcs may be caused by the reflected Alfvén wing, rather than by the main Alfvén wing.

[69] The three Io-controlled radio arcs modeled and fitted here have also been modeled by Hess et al. [2010a]. Figure 6b shows the fits of an Io-B arc (northern hemisphere, Io phase near $90^{\circ}$ ), using the VIPAL and VIP4 model. Although none of these models permits one to reproduce exactly the shape of the observed emissions, the fit by the VIPAL model reaches the same maximum frequency than the observations, whereas the one performed with the VIP4 model does not. Figures $6 \mathrm{c}$ and $6 \mathrm{~d}$ shows the modeling, using the VIPAL model, of an Io-C and an Io-D arcs (southern hemisphere, Io phase near $230^{\circ}$ and $90^{\circ}$, respectively). These two arcs were accurately fitted by the VIP4 model, and are also accurately fitted by the VIPAL model. Since the two models give close results, we do not show the VIP4 modeling for the sake of clarity. The Io-D arc was recorded for an Io's averaged longitude of $94^{\circ}$, i.e., where the surface field predicted by the VIPAL model is the most different from that predicted by the VIP4 model.

[70] Table 4 shows the equatorial lead angles and emitting electron velocities deduced from the fits of the three radio arcs shown in Figure 6. The VIPAL fits give values of the equatorial lead angle consistent (within a few degrees) with the position of the UV spots, whereas those obtained with 
Table 4. Equatorial Lead Angles and Emitting Electron Velocities Deduced From the Fits of the Three Radio Arcs Shown in Figure $6^{\text {a }}$

\begin{tabular}{|c|c|c|c|c|c|c|c|}
\hline \multirow[b]{2}{*}{ Date } & \multirow[b]{2}{*}{ Hemisphere } & \multicolumn{2}{|l|}{ VIPAL } & \multicolumn{2}{|l|}{ VIP4 } & \multirow{2}{*}{$\begin{array}{c}\text { Io's } \\
\text { Average } \\
\text { Longitude }\end{array}$} & \multirow{2}{*}{$\begin{array}{c}\text { VIPAL MAW } \\
\text { Equatorial } \\
\text { Lead Angle }\end{array}$} \\
\hline & & Equatorial Lead Angle & $\mathrm{e}^{-}$Velocity & Equatorial Lead Angle & $\mathrm{e}^{-}$Velocity & & \\
\hline 3 Apr 2008 & north & $-3.8^{\circ}$ & $0.085 \mathrm{c}$ & $-6^{\mathrm{ob}}$ & $0.01 \mathrm{c}^{\mathrm{b}}$ & $242^{\circ}$ & $-1^{\circ}$ \\
\hline 20 Oct 1998 & south & $5.5^{\circ}$ & $0.135 \mathrm{c}$ & $13.6^{\circ}$ & $0.088 \mathrm{c}$ & $263^{\circ}$ & $6^{\circ}$ \\
\hline 27 May 1996 & south & $2^{\circ}$ & $0.19 \mathrm{c}$ & $17.4^{\circ}$ & $0.31 \mathrm{c}$ & $94^{\circ}$ & $3^{\circ}$ \\
\hline
\end{tabular}

${ }^{\mathrm{a}}$ The expected equatorial lead angle, which one should obtain from the VIPAL fits if the emitting field line mapped to the MAW UV spots (black line in Figure 4), is also given.

${ }^{b}$ Values for the best fits, even if the VIP4 model is inaccurate.

the VIP4 model are off by about $10^{\circ}$. Moreover the electron velocity deduced from the fits using the VIPAL model are less variable than those obtained with the VIP4 model. Although one cannot draw conclusion from only three cases, the modeling of the radio arcs using the VIPAL model is consistent with emission above the main UV spot from electrons with a nearly constant (but hemisphere-dependent) energy. This contradicts the conclusions of Hess et al. [2010a], who found that the emissions may occur above the secondary (RAW) spots.

\subsection{Voyager and Pioneer Magnetic Field Measurements}

[71] Figure 8 shows the error on the modeling of the magnetic field measurements of Pioneer 10 and 11 and Voyager 1 and 2 as a function of the $\mathrm{L}$ shell of the magnetic field lines. This error is computed following equation (8). For L shells smaller than 4 Jovian radii, the VIP4 model matches the measurements much better than our model. For larger L shells however, the error on the measurement modeling by the VIPAL model becomes close to that of the VIP4 model, although a bit larger. As the VIP4 model was primarily based on the fit of these measurements, whereas our model is primarily based of the satellite footprint mapping, this result is not surprising. However, the error is close enough to that of the VIP4 model, so that our model can be assumed as a good approximation of the magnetic field in the equatorial plane farther than 4 Jovian radii from the planet. The measurements are well fitted up to $\sim 15 R_{J}$, where the current sheet model of Connerney et al. [1981] is accurate. In order to match farther measurements, other models should be used, such as the Khurana [1997] model.

[72] If we assume that Jupiter's System III(1965) rotation rate is more accurate than the Higgins et al. [1996] rotation rate, the magnetic field should be rotated by $\sim 5^{\circ}$ in longitude. In this case, the fit of the magnetic field by the VIPAL model is slightly better than that of the VIP4 model farther than $10 R_{J}$, i.e., where the dipolar terms dominate. Once again, this suggests that the System III(1965) period is

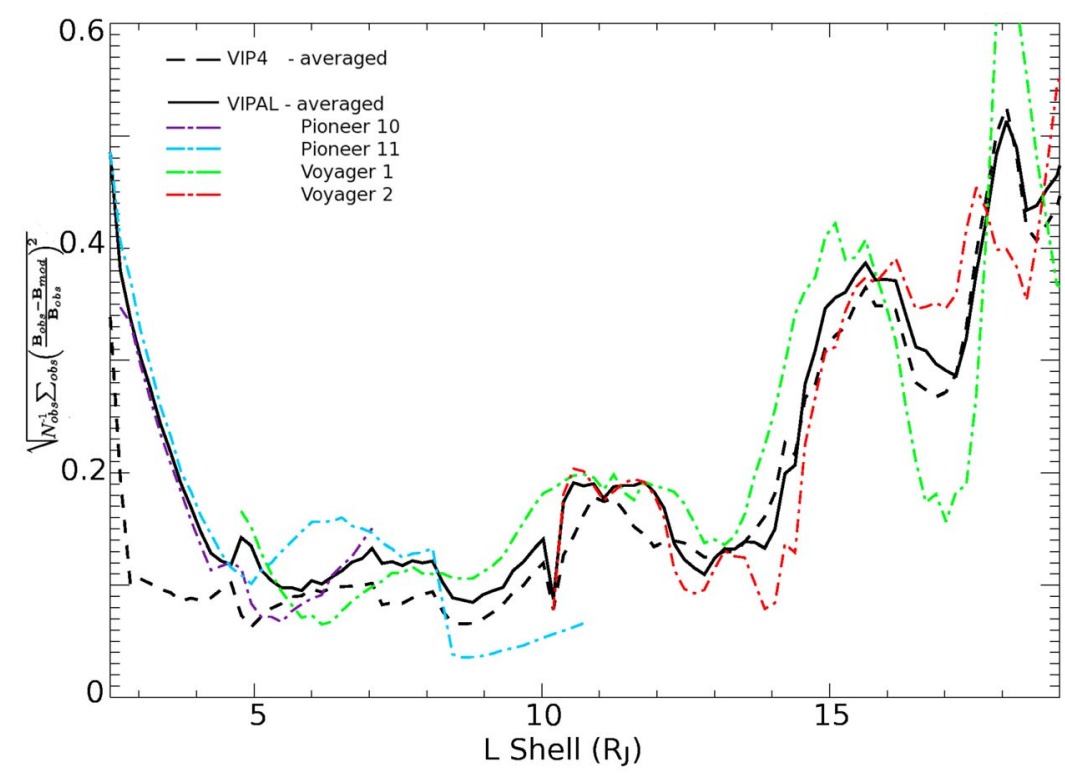

Figure 8. Error on the modeling of the Pioneer 10 and 11 and Voyager 1 and 2 magnetic field measurements, as a function of L shells. Solid line stands for the VIPAL model, and dashed line stands for the VIP4 model, both averaged over all spacecraft observations. VIPAL errors on the modeling of the observations of each spacecraft individually are shown by the dot-dashed lines. The error on the VIPAL model is comparable, although slightly larger than that of the VIP4 model for L shells larger than $4 R_{J}$. 
closer to the Jupiter's period than the Higgins et al. [1996] period.

\section{Discussion}

[73] The VIP4 internal magnetic field model, which we used as a starting point, is a model of fourth degree and fourth order. We chose this model since it fits both the magnetic field measurements and the locations of the Io spots with a reasonably good accuracy, the other models fitting better one of these quantities but worse the other. The study of the error on the lead angle modeling with the VIP4 model showed that this error is important up to the $m=4$ order. In order to correct different $m=4$ lead angle errors in the northern and in the southern hemisphere, it is necessary to use the $g_{5}^{4}$ and $h_{5}^{4}$ coefficients.

[74] In order to be constrained, a model depending on $\mathrm{N}$ coefficients must be constrained by at least $\mathrm{N}$ statistically independent observations. This condition is not verified for all Jovian internal magnetic field models, since the four most constraining flybys give a very sparse sampling of the magnetic field versus longitude and magnetic latitude [Connerney, 1981]. Moreover, the uncertainty on the parameters implies the possibility for several sets of Schmidt coefficients to be in equally good agreement with the data. The 'uniqueness' of the internal magnetic models is achieved by imposing that Schmidt coefficients as small as possible. This additional condition is not physically grounded, but is the most reasonable choice to describe the underdetermined higher orders.

[75] Our model is mostly constrained by the observations of the positions of Io's UV footprints versus Io's orbital longitude. This position is determined every $10^{\circ}$, both in the northern and southern hemisphere, which gives a total of 144 measurements (36 longitude plus 36 latitude measurements per hemisphere). However, we limited ourselves to a fifth-degree/order magnetic field model. Thus, the number of statistically independent measurements is far lower. In each hemisphere, the longitude measurements are defined by five complex Fourier coefficients corresponding to azimuthal numbers $m$ between 1 and 5, since the constant zeroth order is not fitted (see section 4.1). The latitude measurements in each hemisphere are defined by five complex plus one real Fourier coefficients ( $m$ between 0 and 5).

[76] There are five $g_{n}^{0}$ real coefficients, and only two $m=0$ measurements (mean latitudes of the footpaths). There are five $g_{n}^{1}$ and five $h_{n}^{1}$ real coefficients, and eight real (four complex) $m=1$ measurements (longitudes+latitudes). This adds up to five unknown variables.

[77] For higher orders, there are also eight real measurements, but there are eight or less $g_{n}^{m}$ plus $h_{n}^{m}$ real coefficients, so that the higher orders are not underdetermined. This means however, that we have enough spherical harmonics to account in a single manner for the $m>2$ variations of the footprint longitude and latitude, and not that we really have enough data to determine accurately the latitudinal variation of the magnetic field (see above discussion about the relative weight of the UV and magnetometer observations).

[78] Our model would be underconstrained by the UV observations alone, since there are 35 coefficients to determine and only 30 statistically independent UV measurements. Nevertheless, the magnetic field measurements have to be added to this total, since they contribute, albeit in a small proportion, to constraining the model. Thus, even if we cannot ascertain that the model is unique, there are a enough constraints to ensure that all the possible solutions to our fit have similar coefficients. From these possible solutions, the one we selected is determined by the minimization of the amplitude of the Schmidt coefficients that we performed at the end of the process.

[79] However, we showed that the fit of the Io footprints mostly involved the $\left(g_{2}^{0}-g_{4}^{0}\right),\left(g_{2}^{2}-g_{4}^{2}\right)$ and $\left(h_{2}^{2}-g_{4}^{2}\right)$ combinations of Schmidt coefficients. They are, in a fifthdegree internal magnetic field model, the only combinations of Schmidt coefficients able to generate the horseshoe pattern of the northern surface magnetic field strength needed to fit the footprint positions. Nevertheless, these values are not consistent with those deduced from the inversion of the magnetic field measurements by Voyager and Pioneer, and do not provide as a good match as previous models to the magnetic field measurements. This implies that our model is overconstrained: we do not use enough Schmidt coefficients to describe both the Io footprint position and the magnetic field measurements. Hence, it is possible to build a better model with a higher degree, using all the constraints available. Since there are 36 latitude and longitude measurements in each hemisphere, we can show that for any order $m<18$, the Io footprint observations bring eight measurements. Thus the Io footprint observations can constrain four degrees by themselves. Assuming that the magnetic field measurements permit one to constrain the Schmidt coefficients up to the third degree-third order (O6 model [Connerney et al., 1996]), it should be possible to compute a constrained internal magnetic field model of the seventh degree-seventh order, using the Io footprint observations. Such a model may still not be able to describe accurately the magnetic anomaly which requires even higher degrees and order, given its extent of a few tens of degrees both in longitude and latitude.

[80] The computation of such a model would require a very precise study of the dependence of the magnetic field and footprint fits on the Schmidt coefficients, a careful study of the effects of the error on the measurements [Connerney, 1981], and the use of all the observations, including Europa's, Ganymede's footprints and Voyager 2, Ulysses and Galileo measurements, plus a careful analysis of the uniqueness and resolution of the model parameters. A better modeling of the Jovian current sheet would then be necessary. Moreover, it needs the development of a new method to solve the footprint mapping problem. Hence in the present paper, we limited ourselves to a fifth-degree-fifthorder model, which is simpler to constrain and to compute.

\section{Conclusion}

[81] We computed a fifth-degree and fifth-order spherical harmonic decomposition of the Jovian magnetic field by fitting the position of the Io footprints observed in UV.

[82] 1. Our model gives a better description of the positions of Io's, Europa's and Ganymede's footpaths in the northern hemisphere compared to the VIP4 model. The southern satellite footpaths are comparable to those of the VIP4 model. The mean distance between the observed and modeled Io footprints is less than $1^{\circ}$. 
[83] 2. Our model gives an accurate description of the lead angle of the active field line associated with the Io-magnetosphere interaction. The error on the lead angle is about $1^{\circ}$

[84] 3. Our model also gives magnetic field vector along the Voyager and Pioneer trajectories which are consistent with the observations, for $\mathrm{L}$ shells larger than $4 R_{J}$.

[85] 4. Our model gives surface magnetic field strengths along the Io footpath corresponding to electron cyclotron frequencies consistent with the radio observations.

[86] 5. Our model permits a better modeling of the Jovian radio arcs, in particular in the northern hemisphere.

[87] The dipolar moment deduced from the fit of the Io footprint position is shifted by $\sim 5^{\circ}$ relative to the one deduced from the magnetic field measurements. This may be explained by the $\sim 30$ years separation between the magnetic field measurements by the Pioneer and Voyager spacecraft and the Io footprint observations in UV. This $5^{\circ}$ difference can be explained by an underestimation of Jupiter's rotation rate by roughly $25 \mathrm{~ms}$. The IAU rotation rate [Higgins et al., 1996, 1997] used to determine the longitudes of Io and its footprint is $\sim 27 \mathrm{~ms}$ longer than the previous System III (1965) rotation rate. Hence, we suggest that the System III (1965) rotation rate better fits the Jovian rotation rate than the one proposed by Higgins et al. [1996]. The dipolar moment in our model is consistent with the results of Russell et al. [2001a, 2001b] deduced from the Galileo magnetometer measurements.

[88] Acknowledgments. Sébastien Hess acknowledges valuable inputs from F. Bagenal and was supported by the NASA JDAP grants to the University of Colorado at Boulder.

[89] Masaki Fujimoto thanks the reviewers for their assistance in evaluating this paper.

\section{References}

Acuna, M. A., and N. F. Ness (1975), The Pioneer XI high field fluxgate magnetometer, Space Sci. Instrum., 1, 177-188.

Acuna, M. H., and N. F. Ness (1975), Jupiter's main magnetic field measured by Pioneer 11, Nature, 253, 327-328.

Behannon, K. W., M. H. Acuna, L. F. Burlaga, R. P. Lepping, N. F. Ness, and F. M. Neubauer (1977), Magnetic field experiment for Voyagers 1 and 2, Space Sci. Rev., 21, 235-257.

Bigg, E. K. (1964), Influence of the satellite Io on Jupiter's decametric emission, Nature, 203, 1008-1010.

Boischot, A., A. Lecacheux, M. L. Kaiser, M. D. Desch, J. K. Alexander, and J. W. Warwick (1981), Radio Jupiter after Voyager: An overview of the planetary radio astronomy observations, J. Geophys. Res., 86, 8213-8226.

Bonfond, B. (2010), The 3-D extent of the Io UV footprint on Jupiter, J. Geophys. Res., 115, A09217, doi:10.1029/2010JA015475.

Bonfond, B., D. Grodent, J.-C. Gérard, A. Radioti, J. Saur, and S. Jacobsen (2008), UV Io footprint leading spot: A key feature for understanding the UV Io footprint multiplicity?, Geophys. Res. Lett., 35, L05107, doi:10.1029/2007GL032418.

Bonfond, B., D. Grodent, J.-C. Gérard, A. Radioti, V. Dols, P. A. Delamere, and J. T. Clarke (2009), The Io UV footprint: Location, inter-spot distances and tail vertical extent, J. Geophys. Res., 114, A07224, doi:10.1029/ 2009JA014312.

Branduardi-Raymont, G., R. F. Elsner, M. Galand, D. Grodent, T. E. Cravens, P. Ford, G. R. Gladstone, and J. H. Waite Jr. (2008), Spectral morphology of the X-ray emission from Jupiter's aurorae, J. Geophys. Res., 113, A02202, doi:10.1029/2007JA012600.

Burke, B. F., and K. L. Franklin (1955), Observations of a variable radio source associated with the planet Jupiter, J. Geophys. Res., 60, 213-217.

Carr, T. D., M. D. Desch, and J. K. Alexander (1983), Phenomenology of magnetospheric radio emissions, in Physics of the Jovian Magnetosphere, edited by A. J. Dessler, pp. 226-284, Cambridge Univ. Press, Cambridge, U. K.
Clarke, J. T., et al. (1996), Far-ultraviolet imaging of Jupiter's aurora and the Io "footprint," Science, 274, 404-409, doi:10.1126/science.274.5286.404.

Clarke, J. T., et al. (2002), Ultraviolet emissions from the magnetic footprints of Io, Ganymede and Europa on Jupiter, Nature, 415, 997-1000.

Connerney, J. E. P. (1981), The magnetic field of Jupiter: A generalized inverse approach, J. Geophys. Res., 86, 7679-7693, doi:10.1029/ JA086iA09p07679.

Connerney, J. E. P. (1993), Magnetic fields of the outer planets, J. Geophys. Res., 98, 18,659-18,679, doi:10.1029/93JE00980.

Connerney, J. E. P. (2007), Planetary magnetism, in Treatise on Geophysics, vol. 10, Planets and Moons, edited by G. Schubert and T. Spohn, pp. 243-280, Elsevier, Oxford, U. K.

Connerney, J. E. P., and T. Satoh (2000), The $\mathrm{H}_{3}^{+}$ion: A remote diagnostic of the Jovian magnetosphere, in Astronomy, Physics and Chemistry of $H_{3}^{+}$, edited by E. Herbst et al., Philos. Trans. R. Soc. London, Ser. A, 358, pp. 2471-2483.

Connerney, J. E. P., M. H. Acuña, and N. F. Ness (1981), Modeling the Jovian current sheet and inner magnetosphere, J. Geophys. Res., 86, 8370-8384, doi:10.1029/JA086iA10p08370.

Connerney, J. E. P., R. Baron, T. Satoh, and T. Owen (1993), Images of excited $\mathrm{H}_{3}^{+}$at the foot of the Io flux tube in Jupiter's atmosphere, Science, 262, 1035-1038, doi:10.1126/science.262.5136.1035.

Connerney, J. E. P., M. H. Acuña, and N. F. Ness (1996), Octupole model of Jupiter's magnetic field from Ulysses observations, J. Geophys. Res., 101, 27,453-27,458, doi:10.1029/96JA02869.

Connerney, J. E. P., M. H. Acuña, N. F. Ness, and T. Satoh (1998), New models of Jupiter's magnetic field constrained by the Io flux tube footprint, J. Geophys. Res., 103, 11,929-11,939, doi:10.1029/97JA03726.

Delamere, P. A., F. Bagenal, R. Ergun, and Y.-J. Su (2003), Momentum transfer between the Io plasma wake and Jupiter's ionosphere, J. Geophys. Res., 108(A6), 1241, doi:10.1029/2002JA009530.

Ergun, R. E., L. Ray, P. A. Delamere, F. Bagenal, V. Dols, and Y. J. Su (2009), Generation of parallel electric fields in the Jupiter-Io torus wake region, J. Geophys. Res., 114, A05201, doi:10.1029/2008JA013968.

Genova, F., and M. G. Aubier (1985), Io-dependent sources of the Jovian decameter emission, Astron. Astrophys., 150, 139-150.

Gérard, J.-C., A. Saglam, D. Grodent, and J. T. Clarke (2006), Morphology of the ultraviolet Io footprint emission and its control by Io's location, J. Geophys. Res., 111, A04202, doi:10.1029/2005JA011327.

Grodent, D., J.-C. Gérard, J. Gustin, B. H. Mauk, J. E. P. Connerney, and J. T. Clarke (2006), Europa's FUV auroral tail on Jupiter, Geophys. Res. Lett., 33, L06201, doi:10.1029/2005GL025487.

Grodent, D., B. Bonfond, J.-C. Gérard, A. Radioti, J. Gustin, J. T. Clarke, J. Nichols, and J. E. P. Connerney (2008a), Auroral evidence of a localized magnetic anomaly in Jupiter's northern hemisphere, J. Geophys. Res., 113, A09201, doi:10.1029/2008JA013185.

Grodent, D., J.-C. Gérard, A. Radioti, B. Bonfond, and A. Saglam (2008b), Jupiter's changing auroral location, J. Geophys. Res., 113, A01206, doi:10.1029/2007JA012601.

Grodent, D., B. Bonfond, A. Radioti, J.-C. Gérard, X. Jia, J. D. Nichols, and J. T. Clarke (2009), Auroral footprint of Ganymede, J. Geophys. Res., 114, A07212, doi:10.1029/2009JA014289

Hess, S., B. Cecconi, and P. Zarka (2008), Modeling of Io-Jupiter decameter arcs, emission beaming and energy source, Geophys. Res. Lett., 35, L13107, doi:10.1029/2008GL033656.

Hess, S., A. Petin, P. Zarka, B. Bonfond, and B. Cecconi (2010a), Lead angles and emitting electron energies of Io-controlled decameter radio arcs, Planet. Space Sci., 58, 1188-1198, doi:10.1016/j.pss.2010.04.011.

Hess, S. L. G., P. Delamere, V. Dols, B. Bonfond, and D. Swift (2010b), Power transmission and particle acceleration along the Io flux tube, J. Geophys. Res., 115, A06205, doi:10.1029/2009JA014928.

Higgins, C. A., T. D. Carr, and F. Reyes (1996), A new determination of Jupiter's radio rotation period, Geophys. Res. Lett., 23, 2653-2656, doi:10.1029/96GL02388.

Higgins, C. A., T. D. Carr, F. Reyes, W. B. Greenman, and G. R. Lebo (1997), A redefinition of Jupiter's rotation period, J. Geophys. Res., 102, 22,033-22,042, doi:10.1029/97JA02090.

Hill, T. W., and V. M. Vasyliūnas (2002), Jovian auroral signature of Io's corotational wake, J. Geophys. Res., 107(A12), 1464, doi:10.1029/ 2002JA009514.

Jacobsen, S., F. M. Neubauer, J. Saur, and N. Schilling (2007), Io's nonlinear MHD-wave field in the heterogeneous Jovian magnetosphere, Geophys. Res. Lett., 34, L10202, doi:10.1029/2006GL029187.

Khurana, K. K. (1997), Euler potential models of Jupiter's magnetospheric field, J. Geophys. Res., 102, 11,295-11,306, doi:10.1029/97JA00563.

Khurana, K. K., and H. K. Schwarzl (2005), Global structure of Jupiter's magnetospheric current sheet, J. Geophys. Res., 110, A07227, doi:10.1029/2004JA010757. 
Ness, N. F., M. H. Acuna, R. P. Lepping, L. F. Burlaga, K. W. Behannon, and F. M. Neubauer (1979a), Magnetic field studies at Jupiter by Voyager 1: Preliminary results, Science, 204, 982-987.

Ness, N. F., M. H. Acuna, R. P. Lepping, L. F. Burlaga, K. W. Behannon, and F. M. Neubauer (1979b), Magnetic field studies at Jupiter by Voyager 2 : Preliminary results, Science, 206, 966-972.

Neubauer, F. M. (1980), Nonlinear standing Alfvén wave current system at Io: Theory, J. Geophys. Res., 85, 1171-1178.

Prangé, R., D. Rego, D. Southwood, P. Zarka, S. Miller, and W. Ip (1996), Rapid energy dissipation and variability of the Io-Jupiter electrodynamic circuit, Nature, 379, 323-325, doi:10.1038/379323a0.

Queinnec, J., and P. Zarka (1998), Io-controlled decameter arcs and Io-Jupiter interaction, J. Geophys. Res., 103, 26,649-26,666, doi:10.1029/98JA02435

Ray, L. C., and S. Hess (2008), Modelling the Io-related DAM emission by modifying the beaming angle, J. Geophys. Res., 113, A11218, doi:10.1029/2008JA013669.

Russell, C. T., Z. J. Yu, K. K. Khurana, and M. G. Kivelson (2001a), Magnetic field changes in the inner magnetosphere of Jupiter, Adv. Space Res., 28, 897-902, doi:10.1016/S0273-1177(01)00513-0.

Russell, C. T., Z. J. Yu, and M. G. Kivelson (2001b), The rotation period of Jupiter, Geophys. Res. Lett., 28, 1911-1912, doi:10.1029/ $2001 \mathrm{GL} 012917$.

Saur, J. (2004), A model of Io's local electric field for a combined Alfvénic and unipolar inductor far-field coupling, J. Geophys. Res., 109, A01210, doi:10.1029/2002JA009354.

Saur, J., F. M. Neubauer, J. E. P. Connerney, P. Zarka, and M. G. Kivelson (2004), Plasma interaction of Io with its plasma torus, in Jupiter: The Planet, Satellites, and Magnetosphere, edited by F. Bagenal, T. E. Dowling, and W. B. McKinnon, pp. 537-560, Cambridge Univ. Press, Cambridge, U. K.
Thomas, N., F. Bagenal, T. W. Hill, and J. K. Wilson (2004), The Io neutra clouds and plasma torus, in Jupiter: The Planet, Satellites, and Magnetosphere, edited by F. Bagenal, T. E. Dowling, and W. B. McKinnon, pp. 561-591, Cambridge Univ. Press, Cambridge, U. K.

Treumann, R. A. (2006), The electron cyclotron maser for astrophysical application, Astron. Astrophys. Rev., 13, 229-315, doi:10.1007/s00159006-0001-y.

Wu, C. S. (1985), Kinetic cyclotron and synchrotron maser instabilitiesRadio emission processes by direct amplification of radiation, Space Sci. Rev., 41, 215-298.

Zarka, P. (1998), Auroral radio emissions at the outer planets: Observations and theories, J. Geophys. Res., 103, 20,159-20,194, doi:10.1029/ 98JE01323.

Zarka, P. (2004), Fast radio imaging of Jupiter's magnetosphere at lowfrequencies with LOFAR, Planet. Space Sci., 52, 1455-1467.

Zarka, P., J. Queinnec, and F. J. Crary (2001), Low-frequency limit of Jovian radio emissions and implications on source locations and Io plasma wake, Planet. Space Sci., 49, 1137-1149, doi:10.1016/S00320633(01)00021-6.

Zarka, P., D. Langmayr, A. Gerbault, L. Denis, A. Coffre, and F. Korver (2002), Lead angle of Io-controlled radio emission: Alfvén wave or slow shock?, paper presented at Magnetospheres of the Outer Planets Conference, John Hopkins Univ., Columbia, Md

B. Bonfond and D. Grodent, LPAP, Université de Liège, B-4000 Liège, Belgium.

S. L. G. Hess, LASP, University of Colorado at Boulder, Boulder, CO 80303, USA. (sebastien.hess@obspm.fr)

P. Zarka, LESIA, Observatoire de Paris-CNRS, F-92195 Meudon, France. 